\title{
A spectroscopic view on cosmic PAH emission
}

Peeters, E.; Mackie, C.; Candian, A.; Tielens, A.G.G.M.

\section{Citation}

Peeters, E., Mackie, C., Candian, A., \& Tielens, A. G. G. M. (2021). A spectroscopic view on cosmic PAH emission. Accounts Of Chemical Research, 54(8), 1921-1933.

doi:10.1021/acs.accounts.0c00747

Version: $\quad$ Publisher's Version

License: $\quad$ Licensed under Article 25fa Copyright Act/Law (Amendment Taverne)

Downloaded from: https://hdl.handle.net/1887/3263706

Note: To cite this publication please use the final published version (if applicable). 


\title{
A Spectroscopic View on Cosmic PAH Emission
}

\author{
Published as part of the Accounts of Chemical Research special issue "Astrochemistry and Planetary Science". \\ Els Peeters,* Cameron Mackie, Alessandra Candian, and Alexander G. G. M. Tielens
}

Cite This: Acc. Chem. Res. 2021, 54, 1921-1933

Read Online

ACCESS I

Џll Metrics \& More

四 Article Recommendations

CONSPECTUS: Polycyclic aromatic hydrocarbon molecules (PAHs) are ubiquitously present at high abundances in the Universe. They are detected through their infrared (IR) fluorescence UV pumped by nearby massive stars. Hence, their infrared emission is used to determine the star formation rate in galaxies, one of the key indicators for understanding the evolution of galaxies. Together with fullerenes, PAHs are the largest molecules found in space. They significantly partake in a variety of physical and chemical processes in space, influencing star and planet formation as well as galaxy evolution.

Since the IR features from PAHs originate from chemical bonds involving only nearest neighbor atoms, they have only a weak dependence on the size and structure of the molecule, and it is therefore not possible to identify the individual PAH molecules that make up the cosmic PAH family. This

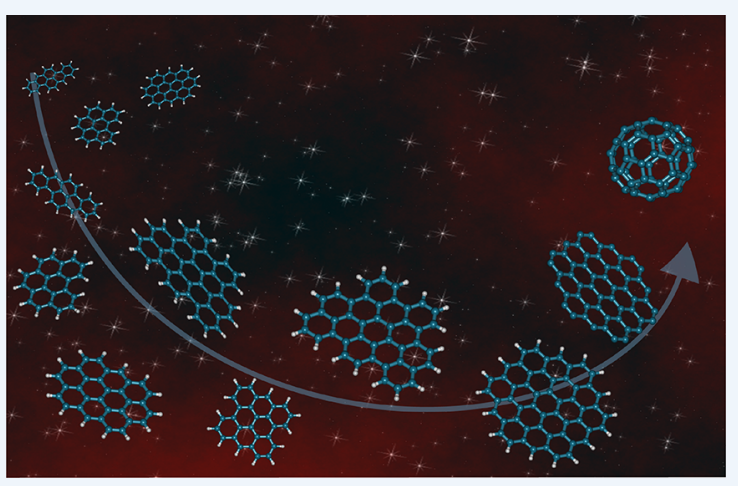
strongly hampers the interpretation of their astronomical fingerprints. Despite the lack of identification, constraints can be set on the characteristics of the cosmic PAH family thanks to a joint effort of astronomers, physicists, and chemists.

This Account presents the spectroscopic properties of the cosmic PAH emission as well as the intrinsic spectroscopic properties of PAHs and astronomical modeling of the PAH evolution required for the interpretation of the cosmic PAH characteristics. We discuss the observed spectral signatures tracing $\mathrm{PAH}$ properties such as charge, size, and structure and highlight the related challenges. We discuss the recent success of anharmonic calculations of PAH infrared absorption and emission spectra and outline the path forward. Finally, we illustrate the importance of models on PAH processing for the interpretation of the astronomical data in terms of the charge balance and PAH destruction.

Throughout this Account, we emphasize that huge progress is on the horizon on the astronomical front. Indeed, the world is eagerly awaiting the launch of the James Webb Space Telescope (JWST). With its incredible improvement in spatial resolution, combined with its complete spectral coverage of the PAH infrared emission bands at medium spectral resolution and superb sensitivity, the JWST will revolutionize PAH research. Previous observations could only present spectra averaged over regions with vastly different properties, thus greatly confusing their interpretation. The amazing spatial resolution of JWST will disentangle these different regions. This will allow us to quantify precisely how PAHs are modified by the physical conditions of their host environment and thus trace how PAHs evolve across space. However, this will only be achieved when the necessary (and still missing) fundamental properties of PAHs, outlined in this Account, are known. We strongly encourage you to join this effort.

\section{KEY REFERENCES}

- Andrews, H.; Candian, A.; Tielens, A. G. G. M. Hydrogenation and dehydrogenation of interstellar PAHs: Spectral characteristics and $\mathrm{H}_{2}$ formation. Astron. Astrophys. 2016, 595, A23. ${ }^{1}$ This work shows, through a detailed model with molecule-specific properties, how the physical conditions in a typical photodissociation region affect the charge and hydrogen coverage of three prototypical PAHs.

- Mackie, C. J.; Candian, A.; Huang, X.; Maltseva, E.; Petrignani, A.; Oomens, J.; Mattioda, A. L.; Buma, W. J.; Lee, T. J.; Tielens, A. G. G. M. The anharmonic quartic force field infrared spectra of five nonlinear polycyclic aromatic hydrocarbons: Benz[a]anthracene, chrysene, phenanthrene, pyrene, and triphenylene. J. Chem. Phys. 2016, 145, 084313. ${ }^{2}$ This work shows the very good agreement between the DFT-based anharmonic spectrum of a few nonlinear PAHs and their experimental highresolution spectra.

Received: November 10, 2020

Published: March 29, 2021 


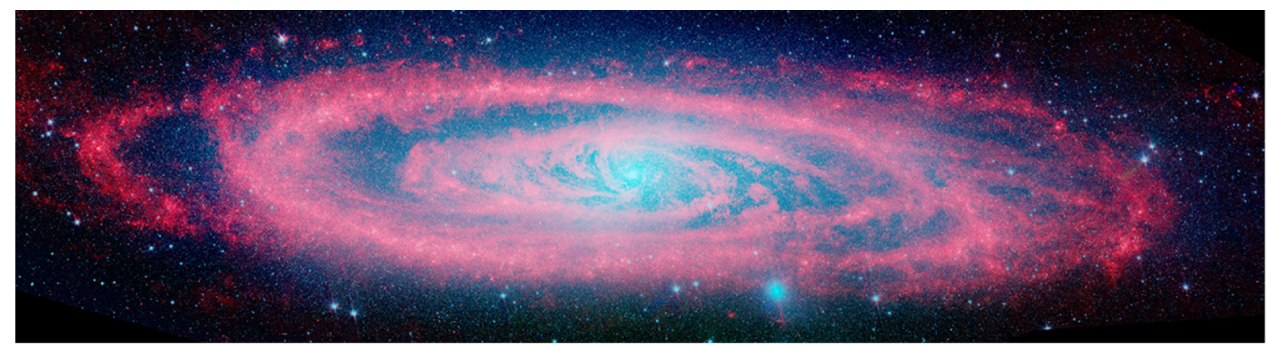

Wavenumber $\left[\mathrm{cm}^{-1}\right]$

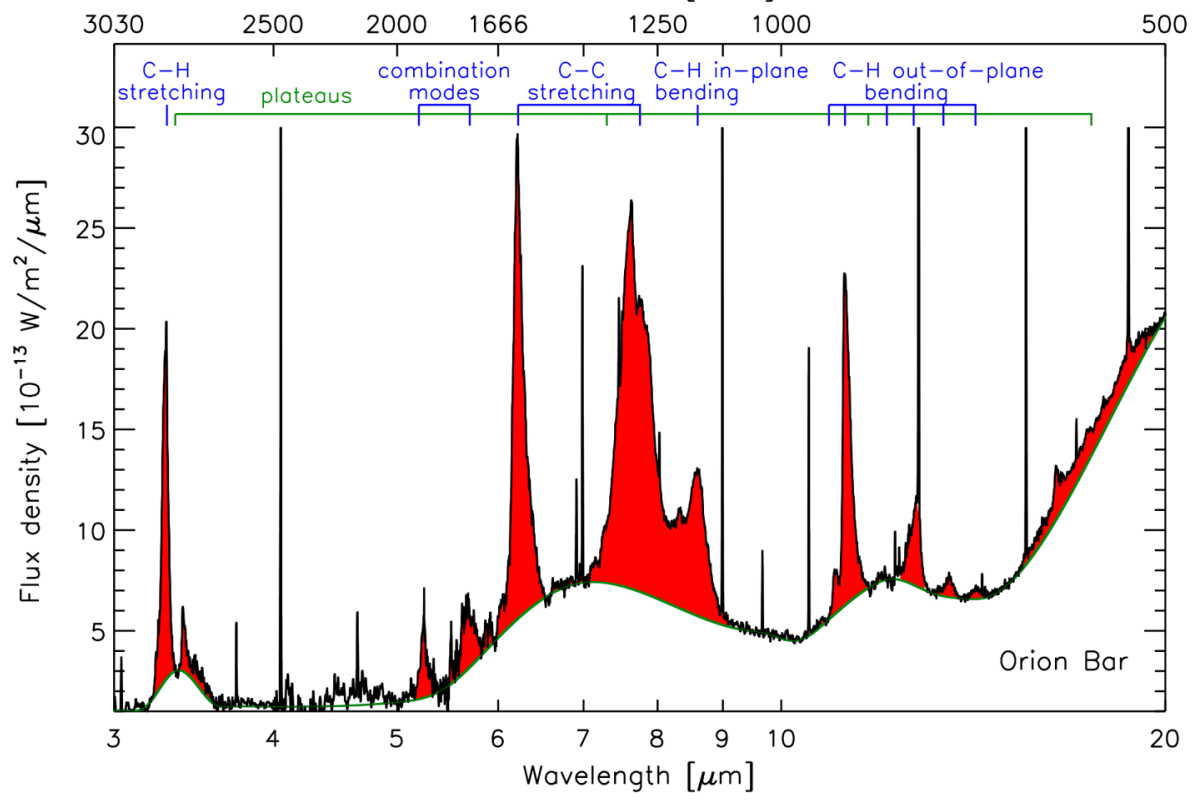

Figure 1. Top: Spitzer-IRAC image of the Andromeda galaxy with the 3.6 and $4.5 \mu \mathrm{m}\left(2778\right.$ and $\left.2222 \mathrm{~cm}^{-1}\right)$ emission (respectively blue and green) representing the stellar population and the $8.0 \mu \mathrm{m}\left(1250 \mathrm{~cm}^{-1}\right)$ emission (red) showcasing the ubiquitous PAH emission. Credit: NASA/ JPL-Caltech/P. Barmby (Harvard-Smithsonian CfA). Bottom: The IR spectrum of the star-forming region, the Orion Bar, observed by the Infrared Space Observatory (ISO). The AIBs are shown in red, superimposed on top of broad plateaus. The corresponding vibrational modes are labeled at the top. Adapted with permission from ref 25. Copyright 2004 Astronomical Society of the Pacific.

- Peeters, E.; Bauschlicher, C. W., Jr.; Allamandola, L. J.; Tielens, A. G. G. M.; Ricca, A.; Wolfire, M. G. The PAH Emission Characteristics of the Reflection Nebula NGC 2023. Astrophys. J. 2017, 836, 198. ${ }^{3}$ This work shows the photochemical evolution of $P A H s$ in the nearby reflection nebula NGC2023.

- Mackie, C. J.; Chen, T.; Candian, A.; Lee, T. J.; Tielens, A. G. G. M. Fully anharmonic infrared cascade spectra of polycyclic aromatic hydrocarbons. J. Chem. Phys. 2018, $149,134302 .{ }^{4}$ In this paper, an infrared cascade emission model based on a Wang-Landau walk is applied to PAHs, where the anharmonicity and resonances of the vibrational spectrum can be fully included.

\section{INTRODUCTION}

The infrared (IR) spectra of objects associated with dust and gas-including the interstellar medium (ISM), star-forming regions, surface layers of proto-planetary disks (the sites of planet formation), and galaxies out to redshifts of $z \sim 4-$ are dominated by strong, broad emission bands (Figure 1). Known collectively as the aromatic infrared bands (AIBs), the main bands are found at 3.3, 6.2, 7.7, 8.6, and $11.2 \mu \mathrm{m}(3030,1612$, 1299,1163 , and $893 \mathrm{~cm}^{-1}$ ) along with a plethora of weaker bands which are perched on top of broad emission plateaus. Multiple carriers have been proposed for the AIBs: hydro- genated amorphous carbon (HAC) ${ }^{6}$ quenched carbon composites (QCCs), ${ }^{7}$ polycyclic aromatic hydrocarbons (PAHs) ${ }^{8,9}$ nanodiamonds, ${ }^{10}$ locally aromatic polycyclic hydrocarbons, ${ }^{11}$ and mixed aromatic-aliphatic organic nanoparticles. ${ }^{12}$ However, this emission is generally attributed to a family of PAH molecules with a typical size of 50-100 C atoms $\left(N_{\mathrm{C}}\right) .{ }^{8,9}$ Upon absorption of a UV photon, PAHs relax by IR fluorescence via their vibrational modes which are detected as the AIBs (Figure 1). The $\mathrm{C}-\mathrm{H}$ stretching mode gives rise to the $3.3 \mu \mathrm{m}\left(3030 \mathrm{~cm}^{-1}\right)$ emission, the $\mathrm{C}-\mathrm{C}$ stretching modes to the 6.2 and $7.7 \mu \mathrm{m}\left(1612\right.$ and $\left.1299 \mathrm{~cm}^{-1}\right)$ emission, the $\mathrm{C}-\mathrm{H}$ in-plane bending mode to the $8.6 \mu \mathrm{m}$ $\left(1163 \mathrm{~cm}^{-1}\right)$ emission, and the $\mathrm{C}-\mathrm{H}$ out-of-plane bending mode to the $10-15 \mu \mathrm{m}\left(1000-666 \mathrm{~cm}^{-1}\right)$ emission. Within the astrophysical context, the PAH family includes PAHrelated species such as, for example, PAHs with side groups, heterosubstituted PAHs, fully or partially (de)hydrogenated PAHs, and PAH clusters (for a definition see ref 13). While aromatic species such as benzene, benzonitrile, and fullerenes $\left(\mathrm{C}_{60}\right.$ and $\left.\mathrm{C}_{70}\right)$ have been detected in space, ${ }^{14-17}$ identification of specific members of this $\mathrm{PAH}$ family (or classes within) remains a mystery.

To date, PAHs are among the largest and most complex molecules known in space. They emit up to $15 \%$ of the total energy output of galaxies and contain $\sim 10 \%$ of the cosmic carbon. PAHs pervade the Universe. PAHs play a crucial role 


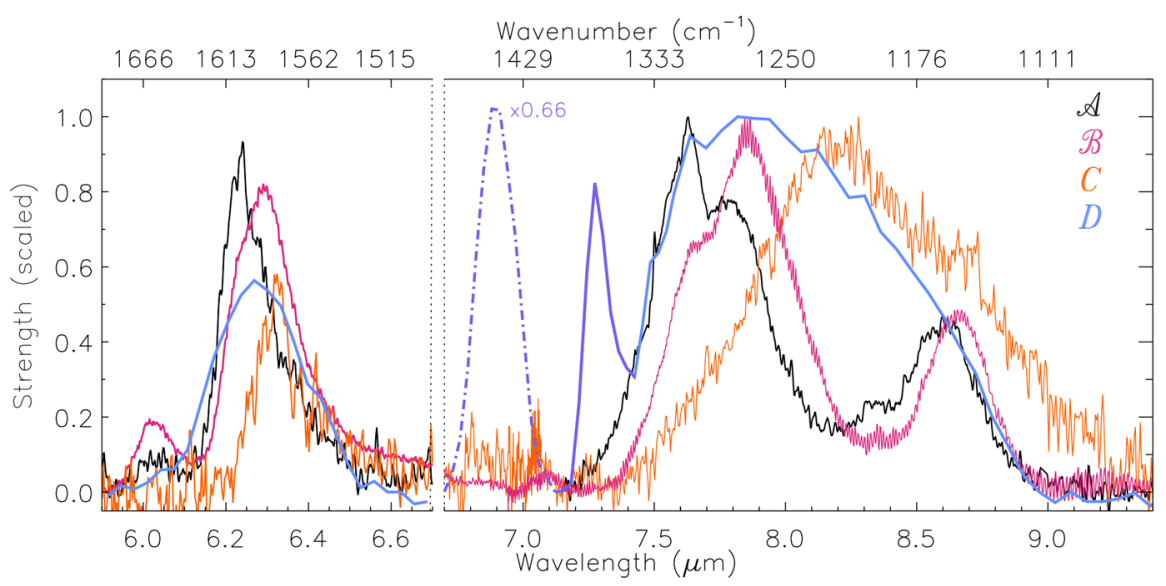

Figure 2. Spectroscopic variation in the 6-9 $\mu \mathrm{m}\left(1666-1111 \mathrm{~cm}^{-1}\right)$ PAH emission. Profiles are normalized to the peak intensity (Jy) in the $8 \mu \mathrm{m}$ $\left(1250 \mathrm{~cm}^{-1}\right)$ region. Strong aliphatic emission at 6.9 and $7.25 \mu \mathrm{m}\left(1449\right.$ and $1379 \mathrm{~cm}^{-1}$, purple) is seen in class $\mathcal{D}$. Class $\mathcal{A}$ is represented by IRAS $23133+6050$, class $\mathcal{B}$ by HD 44179, class $C$ by IRAS 13416-6243, and class $\mathcal{D}$ by IRAS f05110-6616. Adapted with permission from ref 26 . Copyright 2002 EDP Sciences.
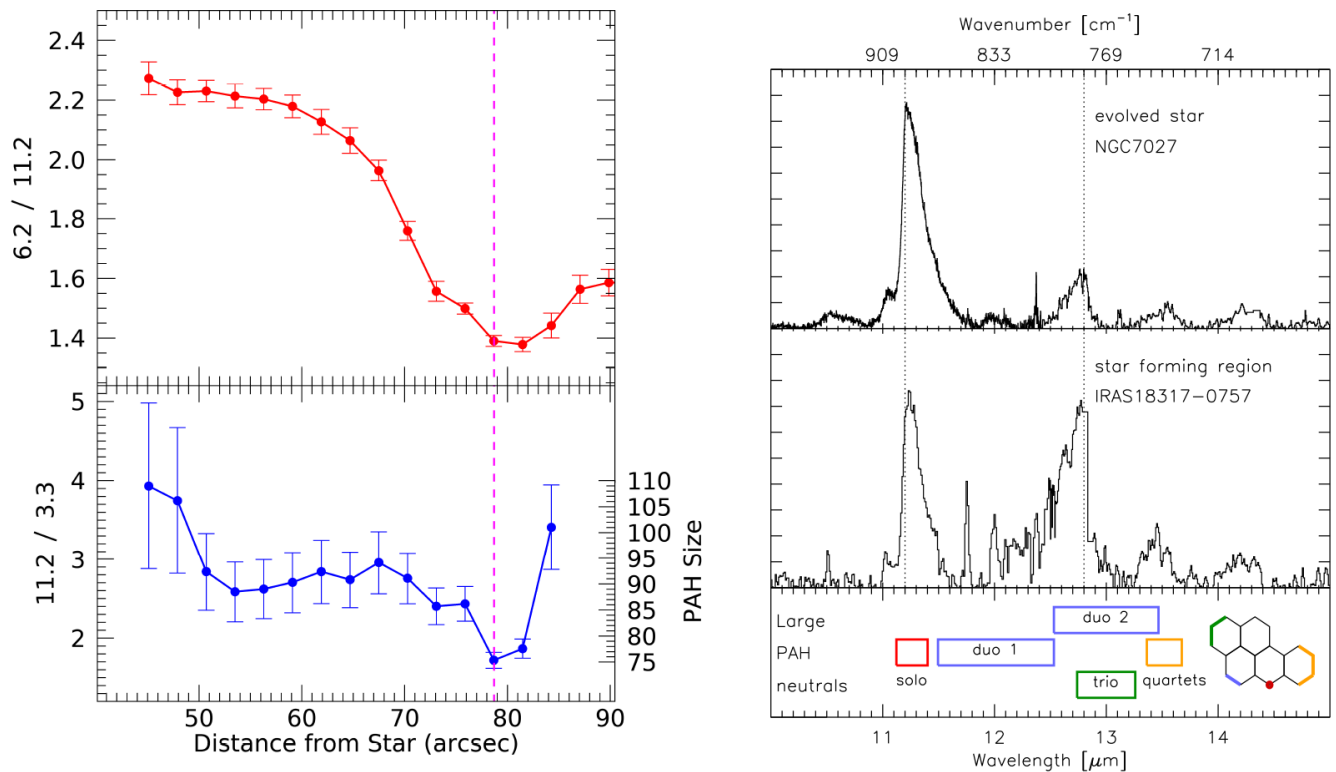

Figure 3. Left: Variation of PAH charge, as traced by the 6.2/11.2 PAH ratio (1612/893, top), ${ }^{3}$ and dominating PAH size, as traced by the 3.3/ 11.2 PAH ratio $\left(3030 / 893\right.$, bottom), ${ }^{57,58}$ as a function of distance from the illuminating star (i.e., UV radiation field strength) in NGC 2023. Adapted with permission from refs 3 and 58. Copyright 2017 AAS and 2020 Collin Knight. Right: Illustrating the observed range in the relative strength of the $\mathrm{CH}$ out-of-plane bending modes (top panels). The bottom panel shows the edge assignment in terms of $\mathrm{CH}$ groups based on harmonic DFT calculations. Adapted with permission from ref 37. Copyright 2009 AAS.

in several astrophysical and astrochemical processes and thus influence star and planet formation. They control the temperature of the gas in the neutral ISM through the photoelectric effect and provide large surface areas for interstellar chemistry. They influence the charge balance and thus molecular abundances in interstellar clouds. In turn, the composition of the $\mathrm{PAH}$ population (e.g., size distribution, charge balance, molecular structure) is influenced by the local physical conditions via photoprocessing, shocks, and formation and destruction processes. A comprehensive understanding of the AIBs thus enables their use as a diagnostic tool of the physical conditions of their host environment (radiation field, and thus stellar population of a galaxy; density; and temperature). ${ }^{18-22}$ They are easily detected in the distant Universe and are used to determine redshifts of distant galaxies. ${ }^{23}$ As they are most efficiently excited by UV radiation, which is abundantly produced by young massive stars, they are used as a tracer for star formation in the Universe. ${ }^{24}$ Furthermore, although PAHs are not found in living cells, they can provide the carbon reservoir necessary to produce complex organic molecules that are building blocks of biological molecules.

Here, we focus on the spectroscopic properties of astronomical PAHs and corresponding intrinsic spectroscopic $\mathrm{PAH}$ properties and $\mathrm{PAH}$ evolution models employed for the interpretation of the astronomical observations. In addition, we highlight the intrinsic $\mathrm{PAH}$ properties yet to be determined in order to fully reap the benefits of the 10 billion dollar investment in the James Webb Space Telescope (JWST). ${ }^{5}$ 


\section{Spectroscopic Properties of Astronomical PAHs}

The cosmic PAH emission is remarkably constant across the diverse environments in which they are observed. Only upon a closer look does the diversity of the cosmic PAH emission become apparent. This diversity is observed in both the relative band strengths and the band profiles for a collection of independent objects and spatially within extended sources.

The profiles of the main PAH bands are distinguished into four classes, $\mathcal{A}, \mathcal{B}, \mathcal{C}$, and $\mathcal{D}$, with the most prominent variations present in the $7.7 \mu \mathrm{m}\left(1299 \mathrm{~cm}^{-1}\right)$ complex (Figure 2). ${ }^{26-29}$ These empirical classifications are primarily based on the peak position of the bands. Moderate to large variations are furthermore seen within class $\mathcal{D}$ and $\mathcal{B}$, respectively, ${ }^{26,30}$ and small changes are detected spatially within extended sources. ${ }^{20,31-33}$ The variety of profiles is the same in the nearby galaxies the Small and Large Magellanic Cloud as in the Milky Way. ${ }^{29,34}$ The classes are known to depend on object type and hence environment. ${ }^{26-28}$ In general, interstellar material exhibits class $\mathcal{A}$ profiles, whereas circumstellar material displays profiles of classes $\mathcal{B}, \mathcal{C}$, and $\mathcal{D}$. Furthermore, for post-AGB stars and Herbig AeBe stars of class $\mathcal{B}$ or $C$ but not class $\mathcal{D}$, the central wavelengths of the main bands show an anticorrelation with the temperature of the exciting star suggesting an origin in UV photoprocessing which influences the characteristics of the PAH population..$^{28,30,35}$ Variations in PAH size, ${ }^{36-39}$ molecular edge structure, ${ }^{3,32}$ charge, $^{3,37}$ fraction of heterosubstituted PAHs (PANHs), ${ }^{26,40}$ and the aliphatic-to-aromatic ratio ${ }^{35,41-45}$ have been put forward to drive the profile variations. Furthermore, the reported correlation between the peak positions of the 6.2 and 7.7 $\mu \mathrm{m}$ (1612 and $\left.1299 \mathrm{~cm}^{-1}\right)$ bands and the $2175 \AA$ bump ${ }^{46}$ provides an interesting link between UV absorption and IR emission properties and warrants further investigations. Thus, while the origin of the profile variations remains unclear, they reveal the structural and/or chemical differences in the PAH family.

Relative PAH intensity variations, in particular among the main bands, are well established. The 3.3 and $11.2 \mu \mathrm{m}$ (3030 and $893 \mathrm{~cm}^{-1}$ ) bands correlate with each other but neither does with the $6.2,7.7,8.6$, or $11.0 \mu \mathrm{m}(1612,1299,1163$, or $909 \mathrm{~cm}^{-1}$ ) bands; the latter four bands instead correlate with each other (Figure 3). ${ }^{47-49}$ Experimental matrix-isolated infrared spectra of PAHs exhibit a similar behavior: neutral PAHs have strong emission at 3.3 and $11.2 \mu \mathrm{m}$ (3030 and 893 $\left.\mathrm{cm}^{-1}\right)$, while cationic PAHs have their strongest emission between 6 and $\left.9 \mu \mathrm{m}\left(1666-1111 \mathrm{~cm}^{-1}\right)\right)^{50,51}$ The PAH charge balance governs the relative strength of the main bands. ${ }^{18}$ Recent observations indicate however a more complicated picture. First, on small spatial scales, the wellknown correlation between 6.2 and $7.7 \mu \mathrm{m}$ (1612 and 1299 $\mathrm{cm}^{-1}$ ) PAH bands breaks down. ${ }^{52,53}$ Second, despite exhibiting the strongest correlations, the cationic bands have distinct spatial morphologies and seem to behave as roughly two distinct groups: the 6.2 and $7.7 \mu \mathrm{m}$ (1612 and $1299 \mathrm{~cm}^{-1}$ ) PAH bands on one hand and the 8.6 and $11.0 \mu \mathrm{m}$ (1163 and $909 \mathrm{~cm}^{-1}$ ) PAH bands on the other hand. ${ }^{3,54}$ Third, the 7.7 $\mu \mathrm{m}\left(1299 \mathrm{~cm}^{-1}\right)$ band is composed of at least two subcomponents with distinct morphologies, one of which is similar to that of the underlying plateau and dust continuum emission. ${ }^{3}$ Finally, the ratio of any two ionic bands depends on the environment. ${ }^{55,56}$ Hence, the ionic bands are due to multiple PAH subfamilies which have their own distinct intrinsic PAH properties. They thus indicate that we do probe changes in PAH family beyond cationic versus neutral, possibly either multiple ionization stages or PAH structure or both. Future observations at high spatial and spectral resolution are needed to fully disentangle and characterize these observational behaviors.

The $\mathrm{PAH}$ size distribution is probed by the relative intensity of the 3.3 and $11.2 \mu \mathrm{m}$ ( 3030 and $893 \mathrm{~cm}^{-1}$ ) band because, upon absorption of a photon, smaller PAHs become more highly excited and thus emit more in high frequency modes than do larger PAHs (as demonstrated by IR fluorescence models applied to harmonic density functional theory (DFT) calculations). ${ }^{8,9,59-61}$ As both bands originate from neutral PAHs, the charge balance is out of play. The dominating PAH size correlates with the strength of the UV radiation field (Figure 3), ${ }^{57,58,62,63}$ indicating that, in stronger UV radiation fields, smaller PAHs are more efficiently destroyed and thus removed from the $\mathrm{PAH}$ family, resulting in an increased (dominating) $\mathrm{PAH}$ size. In low metallicity galaxies, however, there might be other factors controlling the PAH size distribution. $^{64,65}$

The $10-15 \mu \mathrm{m}\left(1000-667 \mathrm{~cm}^{-1}\right)$ region harbors the $\mathrm{CH}$ out-of-plane bending modes whose wavelength depends on the number of adjacent peripheral $\mathrm{C}$ atoms bonded to a $\mathrm{H}$ atom (as demonstrated by both experimental spectra and DFT calculations; Figure 3). ${ }^{66}$ Their relative intensities thus constrain the possible edge structures dominating the 10-15 $\mu \mathrm{m}\left(1000-667 \mathrm{~cm}^{-1}\right)$ PAH emission. ${ }^{37,67}$ These changes are most easily detected by the $11.2 / 12.7 \mathrm{PAH}$ intensity ratio $(893 / 787)$ : the $12.7 \mu \mathrm{m}\left(787 \mathrm{~cm}^{-1}\right)$ band can be completely dwarfed by the $11.2 \mu \mathrm{m}\left(893 \mathrm{~cm}^{-1}\right)$ band or can be equally prominent (Figure 3). Hence, the cosmic PAH family is dominated by large compact PAHs (with many solo $\mathrm{CH}$ groups) in some environments, whereas it exhibits a more irregular edge structure in others. The 12.7/11.2 ratio (787/ 893) also correlates with the charge balance, as traced by, e.g., the $6.2 / 11.2$ ratio $(1612 / 893)$ in space. ${ }^{19,67,68}$ Similarly, harmonic DFT calculations have shown that a changing charge balance also influences the $11.2 / 12.7$ ratio $(787 / 893)$ given that the $11.2 \mu \mathrm{m}\left(893 \mathrm{~cm}^{-1}\right)$ band is dominated by neutral PAHs while both neutral and cationic PAHs contribute to the $12.7 \mu \mathrm{m}\left(787 \mathrm{~cm}^{-1}\right)$ band. ${ }^{36,37,69,70}$ However, to what extent changes in molecular edge structure mimic changes in charge balance is yet to be determined quantitatively. This has important ramifications, though, as the charge balance is rapidly set by the local physical conditions, and this thus may indicate that $\mathrm{PAH}$ processing leading to changes in edge structure occurs on a similar time scale. ${ }^{67}$

In addition to the strong $3.3 \mu \mathrm{m}\left(3030 \mathrm{~cm}^{-1}\right)$ band, weaker emission is commonly observed at $3.4 \mu \mathrm{m}\left(2941 \mathrm{~cm}^{-1}\right)$. This band has been attributed to the aliphatic $\mathrm{CH}$ stretching mode in methylated PAHs or superhydrogenated PAHs. In the interstellar medium, the relative strength of the 3.4/3.3 (2941/ 3030 ) correlates with the strength of the UV radiation field as a consequence of UV photoprocessing (i.e., the preferential destruction of the weaker aliphatic bond). ${ }^{71-74}$ Additional aliphatic bands have been observed at 6.9 and $7.25 \mu \mathrm{m}$ (1449 and $\left.1379 \mathrm{~cm}^{-1}\right) ;^{28,29,75}$ however, these are far less common.

Deuterated hydrocarbons (PADs) have been detected in a handful of sources through the aromatic and aliphatic CD stretching modes at 4.4 and $\sim 4.65 \mu \mathrm{m} \quad(2273$ and 2150 $\left.\mathrm{cm}^{-1}\right) .^{76-78}$ In contrast to the $\mathrm{CH}$ stretching modes, the aliphatic $\mathrm{CD}$ stretching mode is stronger than the aromatic $\mathrm{CD}$ 
stretching mode. As these observations have been challenging, further investigation with JWST is warranted.

As is unequivocally evident from observations, the PAH family in space is not static but undergoes photon-driven processing that alters the characteristics of the species and thus the chemical makeup of the PAH family (toward the end of this Account, we will discuss some of the chemistry affecting PAHs). The Infrared Space Observatory (ISO) revolutionized $\mathrm{PAH}$ research by offering spectra with full wavelength coverage $\left(2-45 \mu \mathrm{m} ; 5000-222 \mathrm{~cm}^{-1}\right)$ at medium spectral resolution. It revealed the spectral richness of PAH emission in all its glory. However, the limited sensitivity and spatial resolution hampered our understanding of the origin of the myriads of substructures present. The Spitzer Space Telescope brought the next revolution owing to its high sensitivity and much improved spatial resolution. It demonstrated the power of spectral imaging to correlate spectral signatures with distinct changes under physical conditions that solidified the realization of $\mathrm{PAH}$ evolution. However, Spitzer lacked the spectral resolution (shortward of $10 \mu \mathrm{m}, 1000 \mathrm{~cm}^{-1}$ ) to reveal the detailed substructure and had no access to the $3.3 \mu \mathrm{m}(3030$ $\mathrm{cm}^{-1}$ ) region crucial to determine PAH sizes. The launch of JWST will bring us the biggest revolution yet, offering access to the full wavelength range of importance for PAH studies at medium spectral resolution and at unprecedented spatial resolution and sensitivity-all at the same time! JWST will spatially resolve, for the first time, the chemical frontiers where the photochemical evolution of PAHs occurs, will enable disentangling of the spectral (sub)components, and will reveal the band profiles while providing a complete description of the physical conditions through molecular and atomic lines, thus revealing the full details of the (photochemical) evolution of the cosmic PAH family. Not only is JWST made for PAH research, the Orion Bar-the prototypical and nearby starforming region rich in $\mathrm{PAH}$ emission (Figure 1) - is among the first targets to be observed by JWST through the Early Release Science program "Radiative Feedback from Massive Stars” (ID1288, led by O. Berné, E. Habart, E. Peeters). ... A $\mathrm{PAH}$ ( $\mathrm{r}$ )evolution is upon us. Interpretation of this treasure trove requires (i) a large database of intrinsic PAH spectra, (ii) an emission model as cosmic PAHs are highly vibrationally excited, and (iii) an astronomical PAH processing model as the observed spectral variations imply that an active chemistry, in particular a photon-driven chemistry, is shaping the cosmic PAH populations.

\section{Intrinsic Spectroscopic Properties of PAHs}

PAH Absorption Spectra. Because of their low volatility, recording $\mathrm{PAH}$ absorption spectra in the gas phase at low temperatures is very challenging. Some spectra at room temperature are available through the NIST database. Absorption spectra of a few typical PAHs were also recorded in the gas phase up to a temperature of $1000 \mathrm{~K}$, with the aim of finding an empirical rule connecting the temperature to the peak and width of the strongest vibrational bands. ${ }^{79}$ The large majority of studies focused on matrix isolation spectroscopy where PAHs are volatilized and then codeposited with an inert gas on a coldfinger. As the species is isolated, UV radiation can be used to ionize the $\mathrm{PAH}$ and record its spectrum even if it can be challenging to separate ion and neutral bands. While due to interaction with the matrix, band positions will shifttypically by a few wavenumbers ${ }^{80}$ - and the profile will broaden, the matrix isolation technique has not only allowed great insight on the intrinsic spectroscopic properties of PAHs but has also provided a solid benchmark for the theoretical calculations. $^{50,69,81-83}$ The spectra of PAH cations can be measured very efficiently using the IR multiphoton dissociation technique (IRMPD ${ }^{84}$ ) where cations are stored in an ion trap and then dissociated when a tunable IR laser is in resonance with an absorption band of the species. The spectrum is then recorded by sweeping the ion-trap into a time-of-flight mass spectrometer. Mass spectrometry is a very sensitive technique, but as absorption of many photons is involved, IRMPD is nonlinear and intensity measurements are comparable to a first order with calculations. Moreover, anharmonicity may shift bands out of resonance, affecting the peak position. The advantage of this technique is the possibility to probe the vibrational spectrum of unusual PAHs, such as protonated ${ }^{85}$ or dehydrogenated ${ }^{86}$ species. In addition, information about the fragmentation and hence the stability of the studied molecules comes for free. Gas-phase spectra of neutral PAHs have been measured in emission using a very sensitive IR detector ${ }^{87,88}$ or in absorption using a messenger technique. ${ }^{89}$ Both techniques are very demanding, and these studies have been limited to a few small PAHs. In recent years, the ion-dip technique has been used to systematically measure IR absorption spectra of small PAHs in the gas phase. ${ }^{90-93}$ It will be important to extend these studies to larger $\mathrm{PAH}$ sizes to validate the theoretical calculations.

In silico studies of the IR spectra of neutral and charged PAHs are generally performed using DFT in the double harmonic approximation, since more accurate methods such as coupled-cluster theory are still prohibitive for such large molecules. Thanks to this method (highly symmetric) species as large as $400 \mathrm{C}$ atoms have been calculated ${ }^{36,37,61,94}$ and the results are available to the community through web-based databases, containing $\sim 4000$ spectra. $^{33,95-99}$ A detailed benchmark study validated the use of the hybrid B3LYP functional in conjunction with the 4-31G basis set to obtain accurate frequencies. ${ }^{98}$ However, the use of a modest size basis-set affects the calculated IR intensities; in particular, the $\mathrm{C}-\mathrm{H}$ stretch intensities are overpredicted by $20 \% .{ }^{99}$ To account for the approximation of the electronic structure calculations and anharmonicity effects, the spectra are "calibrated" by comparison to the matrix isolation spec$\operatorname{tra}^{33,96,97}$ and recently to the gas-phase spectrum of naphthalene. ${ }^{97}$ The spectra are then shifted using a scaling factor. Initially, a uniform scaling factor was applied, estimated to be 0.963 for PAHs, but in recent years, multiple, frequency/ mode-dependent scaling factors have been used. ${ }^{97}$ It is heartening to notice that, after applying multiple scaling factors, calculated harmonic frequencies are in agreement with experiments at the $0.2 \%$ level except in regions of strong resonance effects. While substantial, the database is biased in the number of isomers and charge states and misses systematic studies of dehydrogenated PAHs as well as carbon cages and fullerenes. We strongly emphasize that, while limitations may affect detailed comparison with observed spectra, these theoretical studies allow investigations into systematic trends in shape, size, and chemical composition, reaching reliable conclusions, and are indispensable for our knowledge of the cosmic PAH population. For example, quantum chemistry has demonstrated the importance of the charge state for the relative strength of the $\mathrm{CH}$ versus $\mathrm{CC}$ modes ${ }^{26,99}$ and the edge structure for the pattern of the $\mathrm{CH}$ out-of-plane bending modes. $^{36,37}$ 

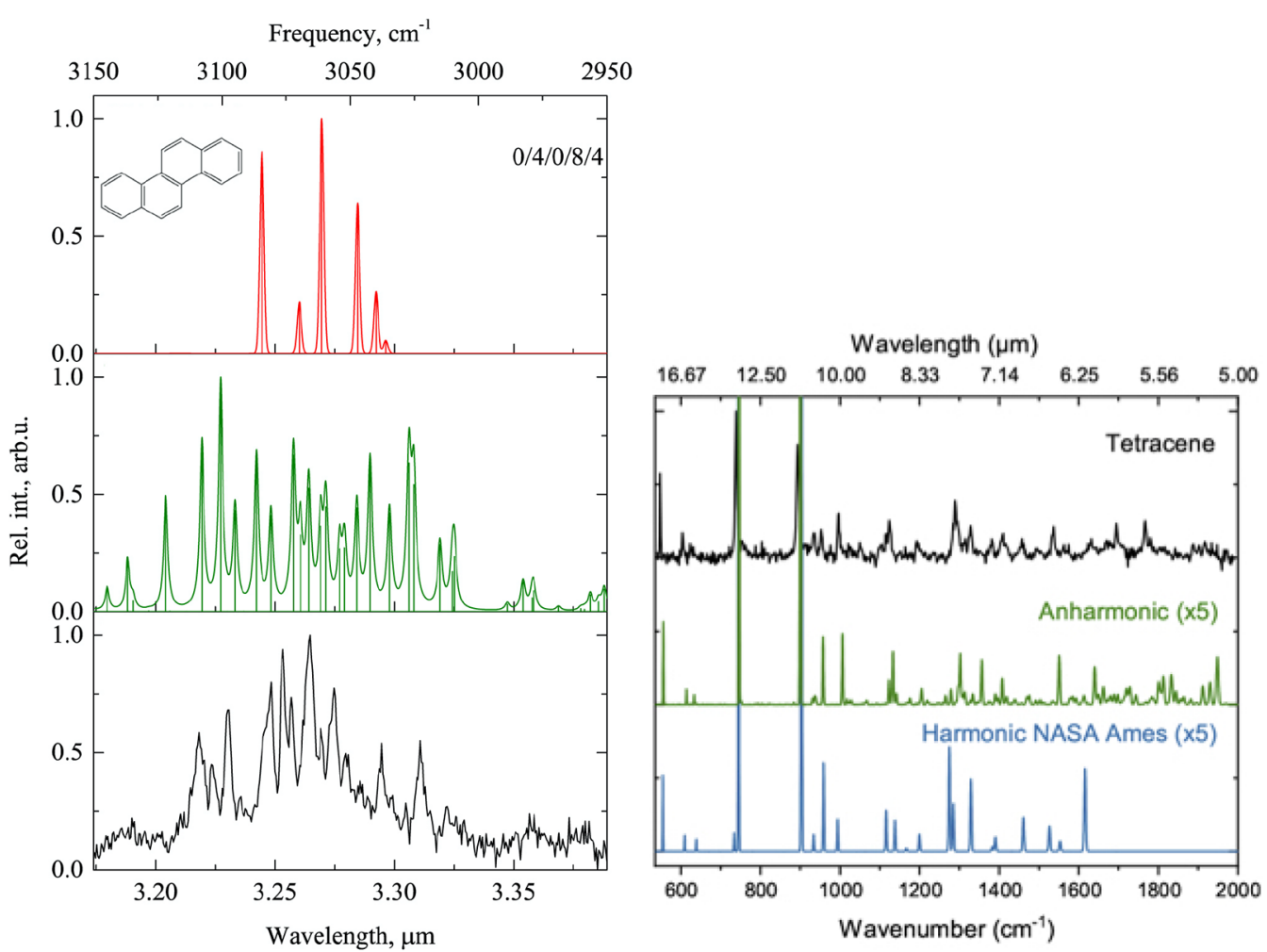

Figure 4. Left: IR absorption spectrum of chrysene in the $3.3 \mu \mathrm{m}\left(3333 \mathrm{~cm}^{-1}\right)$ region: harmonic spectrum (top), anharmonic spectrum (middle), and experimental gas-phase spectrum (at $20 \mathrm{~K}$; bottom). ${ }^{91}$ Right: IR absorption spectrum of tetracene in the $5-17 \mu \mathrm{m}\left(2000-588 \mathrm{~cm}{ }^{-1}\right)$ region: experimental gas-phase spectrum (at $20 \mathrm{~K}$; top), anharmonic spectrum (middle), and harmonic spectrum (bottom). ${ }^{93}$ Reproduced with permission from refs 91 and 93. Copyright 2016 AAS and 2019 EDP Sciences.

Recently, calculations of the quartic force field in the vibrational potential and the dipole surface of small PAHs within the framework of DFT have been performed. The frequencies and IR intensities can then be evaluated using vibrational perturbation theory (VPT2) where resonances are explicitly taken into account. The bottleneck of these calculations is the evaluation of the third and fourth derivatives of the potential energy surfaces through the finite differences method. Thus, even using a hybrid functional such as B3LYP and a double- $\zeta$ basis set (N07D), only molecules up to five rings are computationally feasible. Figure 4 compares the calculated anharmonic spectra B3LYP/N07D with measured ion-dip spectra in the $3000 \mathrm{~cm}^{-1}(3.3 \mu \mathrm{m})$ and $500-2000$ $\mathrm{cm}^{-1}(5-20 \mu \mathrm{m})$ frequency ranges. ${ }^{2,91,93}$ As there are no scaling factors involved in the anharmonic approach, the results compare very favorably. In the $500-2000 \mathrm{~cm}^{-1}$ range $(5-20 \mu \mathrm{m})$, the peak positions are in very good agreement. We specifically draw attention to the $1700-2000 \mathrm{~cm}^{-1}$ frequency range $(5.0-5.9 \mu \mathrm{m})$ where bands are due to overtone and combination bands of the $\mathrm{CH}$ out-of-plane bending mode. Harmonic calculations show no IR activity, but in the anharmonic approach, these bands become weakly active. The $3000 \mathrm{~cm}^{-1}(3.3 \mu \mathrm{m})$ range is host to the $\mathrm{CH}$ stretching modes and-as there are many similar modes-they are very susceptible to resonance effects that are very sensitive to small frequency errors. The anharmonic calculations are in very good agreement with the experimental spectra in terms of the number of modes and the frequency range over which they are active (Figure 4). In this respect, the calculated IR spectra in the harmonic approximation have clear limitations, even after applying a scaling factor (amounting to $\simeq 100 \mathrm{~cm}^{-1}$ ).
Overall, these anharmonic studies demonstrate that calculated frequencies agree with the measurements at the $0.2 \%$ level without the need for scaling factors and, in addition, they reproduce the relative strength better. ${ }^{2,93,100,101}$ Nevertheless, there is room for improvement, as measurements show that triple combination bands should be accounted for as well ${ }^{90}$ and an accurate benchmark is needed to improve on anharmonic infrared intensities and prediction of symmetric modes. ${ }^{86}$ In this assessment, we should keep in mind that quantum chemical calculations are now approaching the reliability demanded by astronomical observations with the JWST spectrometers, $1-2 \mathrm{~cm}^{-1}$ spectral resolution. For now, computations are limited to small PAHs and it will be important to develop reliable extrapolations into the larger size regime thought to be relevant for the interstellar PAH family. A possible way through is the use of classical molecular dynamics (MD) trajectories, where the energy is calculated on-the-fly using DFT or tight-binding (TB)-DFT. In this approach, the anharmonic vibrational spectrum is obtained as the Fourier transform of the dipole time-autocorrelation function. ${ }^{102}$ In addition, it is prudent to develop general rules to correct harmonic calculated spectra for anharmonicity effects and apply them to the existing (harmonic) $\mathrm{PAH}$ databases. This will greatly magnify the available anharmonic PAH spectra which is needed for the interpretation of the cosmic PAH emission. The fact that, after applying multiple scaling factors, calculated harmonic frequencies are in agreement with experiments at the $0.2 \%$ level except in regions of strong resonance effects suggests that (sub)class-dependent algorithms might be very successful. 


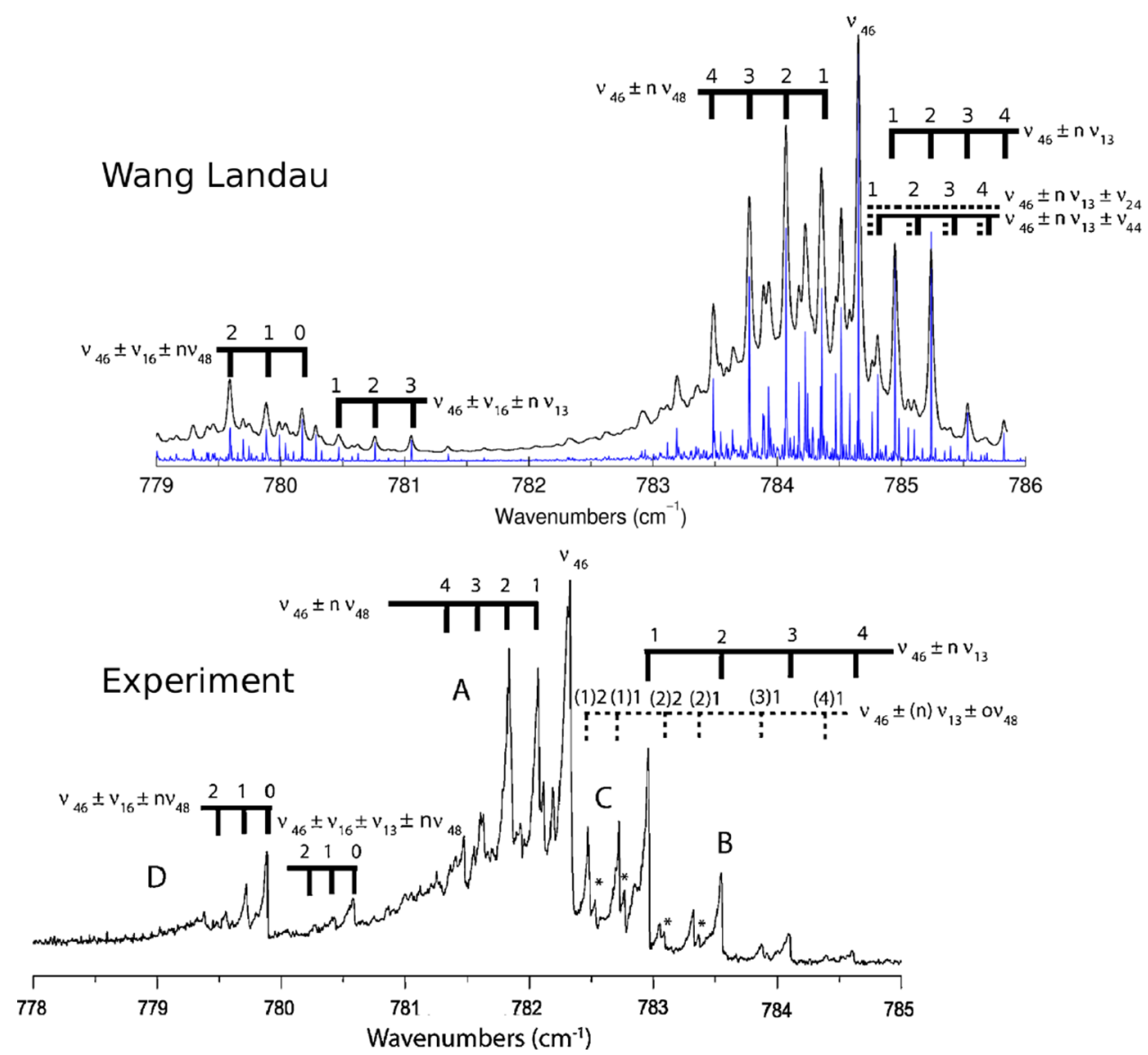

Figure 5. Measured absorption spectrum of naphthalene (at $300 \mathrm{~K}$; bottom ${ }^{104}$ ) compared to the calculated spectrum in the anharmonic approximation (blue sticks) convolved with a $0.02 \mathrm{~cm}^{-1}$ Gaussian (at $300 \mathrm{~K}$; top ${ }^{4}$ ). Anharmonic interactions of the fundamental $\left(\nu_{46}\right)$ with specific modes result in "satellite" bands shifted to lower or higher frequency. Reproduced with permission from refs 4 and 104 . Copyright 2018 and 2009 AIP Publishing.

PAH Emission Spectra. The AIBs are due to UV-pumped IR fluorescence by large gaseous PAHs with typical excitation temperatures of 500-1000 K, depending on size and absorption energy $\left(T \simeq 2000\left(E(\mathrm{eV}) / N_{\mathrm{C}}\right)^{0.4}\right){ }^{103}$ Measured and calculated spectra are however obtained in absorption at $\simeq 0-20 \mathrm{~K}$, and a model for the emission process is required to analyze observed spectra in terms of the molecular characteristics. Specifically, due to anharmonic interactions, peak positions will shift, typically redward, and broaden. The interaction of two vibrational modes, $k$ and $l$, is caught in the anharmonic interaction coefficient $\chi_{k l}$, that describes the shift in the fundamental due to interaction with other (excited) modes; e.g., the total energy is

$$
\frac{E(\vec{n})}{c}=\sum_{k} \nu_{k}\left(n_{k}+1 / 2\right)+\sum_{k \leq l} \chi_{k l}\left(n_{k}+1 / 2\right)\left(n_{l}+1 / 2\right)
$$

The frequency corresponding to the transition $n_{k}-1 \rightarrow n_{k}$ is then given by

$$
\nu(\vec{n})=\nu_{k}+2 \chi_{k k} n_{k}+\sum_{l \neq k} \chi_{k l}\left(n_{l}+1 / 2\right)
$$

where $\vec{n}$ is the vector describing the population of the modes. Figure 5 compares the measured absorption spectrum of naphthalene ${ }^{104}$ with the theoretical spectrum-calculated using the Wang-Landau algorithm and taking these anharmonic interactions into account. The shift in the fundamental mode, $\nu_{46}$, due to anharmonic interactions with specific modes is quite apparent. While there is a small mismatch $\left(\simeq 3 \mathrm{~cm}^{-1}\right)$ in peak frequency of the zero temperature position of the fundamental, the pattern of the anharmonic interactions is well reproduced. Note that there are no free parameters in this comparison.

The magnitude of the frequency shifts due to anharmonic interaction depends on the population of the modes and hence the excitation of the emitting species (Figure 6). In a laboratory setting, emission will occur at a fixed internal energy. Comparisons of the temperature-dependent band position shifts between anharmonic calculations ${ }^{4}$ and experimental measurements ${ }^{79}$ reveal excellent agreement in the temperature coefficients $\left(\chi^{\prime}\right)$ for the $3.3 \mu \mathrm{m}$ feature, while the 8.5 and $12 \mu \mathrm{m}$ features show the theoretical derived values for $\chi^{\prime}$ to be approximately half of the experimental values (see also ref 105 for additional comparisons).

However, observed spectra of interstellar PAHs will be the integrated emission following the full energy cascade from the maximum energy $(\simeq 12 \mathrm{eV})$ immediately after absorption of a UV photon to "zero" internal energy. This energy cascade will lead to a pronounced red shaded wing (Figure 6).,106 Such wings are common characteristics of many AIBs. ${ }^{26,27,107}$ The resulting broadening is quite substantial, and for most bands, an accuracy of a few wavenumbers in calculated peak positions may well be sufficient for a proper comparison with measured AIB spectra. However, accurate profiles will require an 


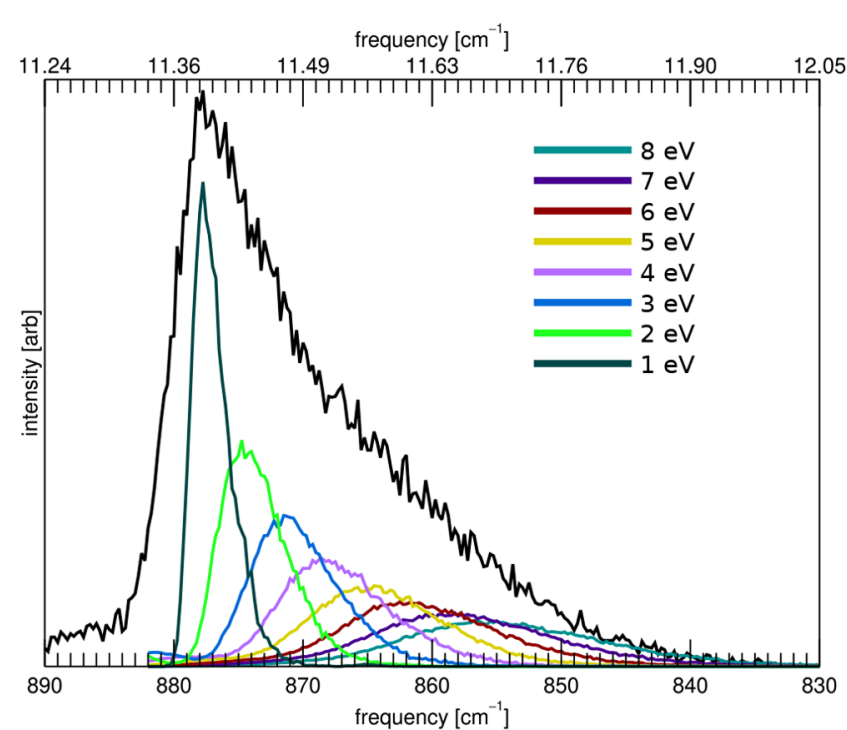

Figure 6. Calculated emission spectrum of the $\mathrm{CH}$ out-of-plane bending mode of anthracene $\left(\mathrm{C}_{14} \mathrm{H}_{10}\right)$ as a function of the excitation energy (colored traces). The black trace is the spectrum calculated following the full energy cascade.

appropriate treatment of the anharmonic interaction between the different modes. Hence, either we have to follow Moore's law or we need to develop clever algorithms that allow anharmonic extrapolations.

PAH Processing. The interstellar PAH family will contain a variety of molecules in a number of ionization stages, and the AIBs will be the sum of the emission from these individual species. The abundance of a species will depend sensitively on the local physical conditions, reflecting the ionization balance and photochemical processing ${ }^{1,108}$ and, in turn, affecting the chemistry.

The ionization balance is a competition between photoionization and electron recombination and is regulated by the ionization parameter, $G_{0} T^{1 / 2} / n_{\mathrm{e}}$ (with $G_{0}$ being the strength of the UV radiation field, $T$ the temperature, and $n_{\mathrm{e}}$ the electron density), which is proportional to the ratio of the ionization rate over the electron recombination rate. ${ }^{109}$ Photoionization potentials and cross sections have been measured for only a number of ionization stages of a limited number of PAHs. ${ }^{110-113}$ Mostly, ionization potentials are estimated using either DFT calculations for specific species or generic rules based upon electrostatic theory for small disks. Electron recombination rates have been measured only for very few (small) PAHs, and these are also generally approximated using electrostatic theory. ${ }^{109}$ The models show that PAHs are mostly neutral in diffuse clouds but in photodissociation regions (PDRs) cations and dications can become the dominant charge carriers. $^{109,114}$

PAHs facilitate charge exchange, and thus, their presence in PDRs influences the abundance of neutral atomic species, such as carbon, sulfur, and silicon. ${ }^{115}$ In addition, in dense clouds, small (30-50 C atoms) PAHs are the major sources of negative charges and, when they are included in models, they trigger an enhancement in the abundance of carbon chains and sulfur-bearing species. ${ }^{116,117}$

PAHs can undergo a strong photochemical evolution in the ISM, weeding out the weakest members of the PAH family. The strong FUV field in PDRs will highly excite the PAHs, and, while IR fluorescence is generally the dominant relaxation channel, fragmentation may also occur when the energy is high enough. Laboratory studies show that the appearance potential for loss of $\mathrm{H}$ or $\mathrm{H}_{2}$ is about $12 \mathrm{eV}$ for coronene $\left(\mathrm{C}_{24} \mathrm{H}_{12}\right)^{118}$ and slightly higher for loss of $\mathrm{C}_{2} \mathrm{H}_{2}$ and thus for coronene (or smaller species) absorption of a single photon can lead to fragmentation. This appearance energy will increase with the size of the species, as it is regulated, to first order, by $E /(3 N-$ 6). ${ }^{119}$ Hence, multiphoton absorption is required for fragmentation of large PAHs. Moreover, the small difference in appearance potential between $\mathrm{H} / \mathrm{H}_{2}$ loss and $\mathrm{C}_{2} \mathrm{H}_{2}$ loss will be greatly amplified for large $\mathrm{PAHs}$ and indeed experiments show that PAHs larger than $30 \mathrm{C}$ atoms are first stripped of all their $\mathrm{H}$ before $\mathrm{C}$ loss sets in (in the form of $\mathrm{C}_{2}$ loss). ${ }^{120}$ As the surface zone of a PDR has a high atomic $\mathrm{H}$ abundance, the $\mathrm{H}$ coverage of a PAH becomes a balance between photochemical loss and reaction with $\mathrm{H}$ and is regulated by $G_{0} / n_{\mathrm{H}}$ with $G_{0}$ being the strength of the UV radiation field and $n_{\mathrm{H}}$ the atomic hydrogen density. This balance is very sensitive to $G_{0} / n_{\mathrm{H}}$, and the transition between PAHs and their completely dehydrogenated products is very sharp (Figure 7 ). ${ }^{1}$ Once a PAH has
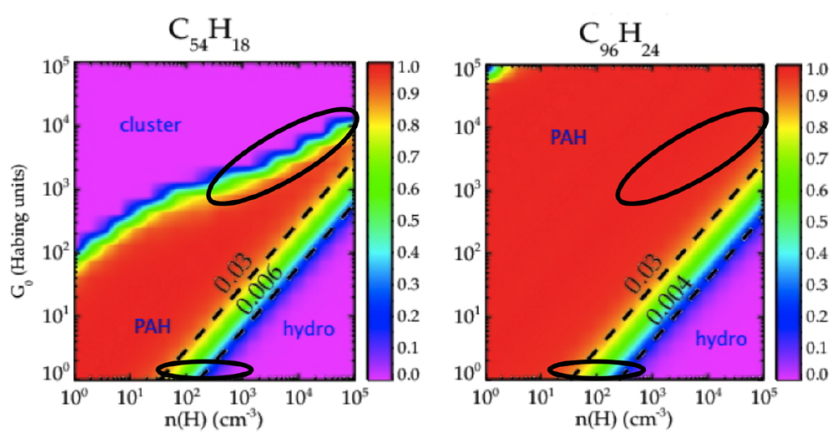

Figure 7. Characteristics of circumcoronene $\left(\mathrm{C}_{54} \mathrm{H}_{18} ;\right.$ left $)$ and circumcircumcoronene $\mathrm{C}_{96} \mathrm{H}_{24}$; right) as a function of the strength of the FUV field, $G_{0}$, and the atomic $\mathrm{H}$ density, $n_{\mathrm{H}}$. For high FUV fields, PAHs will be quickly completely dehydrogenated (labeled clusters), while, for high atomic $\mathrm{H}$ densities, $\mathrm{PAHs}$ will be superhydrogenated (labeled hydro). The two ovals in each panel indicate typical conditions in PDRs (top oval) and in diffuse interstellar clouds (bottom oval). Dashed black lines indicate constant $G_{0} / n_{\mathrm{H}}$ ratios. $^{1}$ Reproduced with permission from ref 1 . Copyright 2016 EDP Sciences.

been stripped of all its H's, C-loss will follow and the resulting cluster may be rapidly destroyed given the increased internal excitation for a given photon energy. However, fragmentation of carbon clusters competes with isomerization to cages and fullerenes which are more stable under these conditions. ${ }^{121,122}$

For high $\mathrm{H}$ densities and low FUV fields, a PAH may react to form superhydrogenated $\mathrm{PAHs}^{1,108}$ which have distinct spectral signatures at 3.4 and $6.85 \mu \mathrm{m}\left(2941\right.$ and $\left.1460 \mathrm{~cm}^{-1}\right)$ due to the presence of $\mathrm{sp}^{3}$ carbon. ${ }^{8,82,123}$ The abundance of superhydrogenated PAHs has also been calculated for two representative interstellar PAHs (Figure 7), revealing that PAHs in PDRs will be normally hydrogenated at best. ${ }^{1}$ The presence of the $3.4 \mu \mathrm{m}\left(2941 \mathrm{~cm}^{-1}\right)$ aliphatic feature in PDRs ${ }^{72,74}$ can then be explained by the presence of PAHs with $\mathrm{CH}_{3}$ groups, or particularly stable forms of superhydrogenated PAHs. ${ }^{124,125}$ Superhydrogenated PAHs can also be important in diffuse interstellar clouds.

As this model illustrates, large PAHs will be very stable in PDRs but smaller PAHs can be destroyed under high FUV 
conditions. For large enough PAHs, isomerization will be a viable pathway toward $\mathrm{C}_{60} \cdot{ }^{121,126}$ Cages and fullerenes are much more stable and may survive better under these conditions. In diffuse interstellar clouds, the high abundance of atomic $\mathrm{H}$ serves to protect PAHs against photofragmentation but $\mathrm{PAHs}$ will acquire additional $\mathrm{H}$ atoms.

In general, comparison of the observed AIBs with model spectra will have to take these aspects into account. Such an analysis will require accurate laboratory spectra of PAHs and their dehydrogenated and superhydrogenated derivatives as well as the kinetic parameters involved in their photoionization, electron recombination, photofragmentation, and isomerization processes. Similar data is needed for carbon cages, fullerenes, clusters of PAHs, and very small grains.

\section{CONCLUDING REMARKS}

The cosmic PAH emission shows clear variability in relative band intensities and band profiles. Largely based on harmonic calculations of intrinsic PAH spectra and experimental data of PAHs and carbonaceous dust samples, these variations are attributed to changing chemical structures, charge balance, and size distribution of the PAH family. This, in turn, has been linked to the changing physical conditions and history of the PAHs' host environment. We are thus able to probe the evolution of the cosmic PAH family. However, while large progress has been made, the origin of the cosmic variations is largely qualitative and often limited by the fact that multiple parameters (e.g., charge, size, composition) change simultaneously.

To improve our interpretation of the astronomical data, intrinsic PAH emission spectra and astronomical PAH models are required. Recently, a major breakthrough was achieved with the first anharmonic intrinsic PAH spectra and their corresponding emission spectra. This anharmonic approach improves dramatically on the harmonic approach in that it better predicts the position and intensity of the fundamental bands and incorporates combination bands, overtones, and hot-bands. Additional work is required to further fine-tune these calculations and to apply them to a larger sample of PAHs with sizes more representative to those present in space. Additional fundamental data is needed for the astronomical modeling of the PAH family. This includes dissociation energies (and channels), isomerization energies, relaxation rates, reaction rates (e.g., with $\mathrm{C}^{+}$), e- recombination rates, $\mathrm{UV}$ absorption cross sections, ionization cross sections, and potentials.

With its unprecedented capabilties, JWST will provide a treasure trove of information on the photochemical evolution of cosmic PAHs at the spatial scale on which PAH processing occurs. JWST will thus significantly relocate the astronomical boundary. Similar progress is then required on the intrinsic and fundamental $\mathrm{PAH}$ properties for the interpretation of these JWST observations. Indeed, to obtain a complete description of the photochemical evolution of PAHs in space, strong collaborations between astronomers, physicists, and chemists is a fundamental necessity.

\section{AUTHOR INFORMATION}

\section{Corresponding Author}

Els Peeters - Department of Physics \& Astronomy and Institute for Earth and Space Exploration, University of Western Ontario, London, Ontario, Canada; SETI Institute,
Mountain View, California 94043, United States;

○ orcid.org/0000-0002-2541-1602; Email: epeeters@

uwo.ca

\section{Authors}

Cameron Mackie - Lawrence Berkeley National Laboratory, University of California, Berkeley, California 94720, United States

Alessandra Candian - van't Hoff Institute for Molecular Sciences, University of Amsterdam, Amsterdam, The Netherlands; Leiden Observatory, Leiden University, Leiden, The Netherlands

Alexander G. G. M. Tielens - Leiden Observatory, Leiden University, Leiden, The Netherlands; University of Maryland, College Park, Maryland 20742, United States

Complete contact information is available at: https://pubs.acs.org/10.1021/acs.accounts.0c00747

\section{Notes}

The authors declare no competing financial interest.

\section{Biographies}

Els Peeters is a professor at the University of Western Ontario and scientist at the SETI Institute. Her research focuses on the physics and chemistry of interstellar carbonaceous molecules and dust.

Cameron Mackie is a postdoctoral scholar at Lawrence Berkeley National Laboratory. His research focus is on theoretical chemistry, from anharmonic infrared spectroscopy (past) to electronic structure theory method development (current).

Alessandra Candian is a research assistant professor at the University of Amsterdam. She researches polycyclic aromatic hydrocarbons and other carbonaceous molecules of astrochemical interest.

Alexander G. G. M. Tielens is a professor at Leiden University and adjunct professor at the University of Maryland. He has significantly contributed to the physics and chemistry of the interstellar medium.

\section{ACKNOWLEDGMENTS}

E.P. gratefully acknowledges sustained support from the Natural Sciences and Engineering Research Council of Canada (NSERC: Discovery grant RGPIN-2020-06434). A.C. thanks the Dutch Science Agency, NWO, for a VENI grant (number 639.041.54). Studies of interstellar chemistry at Leiden Observatory are supported through a grant by the Dutch Science Agency, NWO, as part of the Dutch Astrochemistry Network and through the Spinoza premie from the Dutch Science Agency, NWO.

\section{REFERENCES}

(1) Andrews, H.; Candian, A.; Tielens, A. G. G. M. Hydrogenation and dehydrogenation of interstellar PAHs: Spectral characteristics and $\mathrm{H}_{2}$ formation. Astron. Astrophys. 2016, 595, A23.

(2) Mackie, C. J.; Candian, A.; Huang, X.; Maltseva, E.; Petrignani, A.; Oomens, J.; Mattioda, A. L.; Buma, W. J.; Lee, T. J.; Tielens, A. G. G. M. The anharmonic quartic force field infrared spectra of five nonlinear polycyclic aromatic hydrocarbons: Benz[a] anthracene, chrysene, phenanthrene, pyrene, and triphenylene. J. Chem. Phys. 2016, 145, 084313.

(3) Peeters, E.; Bauschlicher, C. W., Jr.; Allamandola, L. J.; Tielens, A. G. G. M.; Ricca, A.; Wolfire, M. G. The PAH Emission Characteristics of the Reflection Nebula NGC 2023. Astrophys. J. 2017, 836, 198. 
(4) Mackie, C. J.; Chen, T.; Candian, A.; Lee, T. J.; Tielens, A. G. G. M. Fully anharmonic infrared cascade spectra of polycyclic aromatic hydrocarbons. J. Chem. Phys. 2018, 149, 134302.

(5) James Webb Space Telescope. https://webbtelescope.org and https://www.stsci.edu/jwst.

(6) Duley, W. W.; Williams, D. A. A 3.4 micron absorption band in amorphous carbon Implications for interstellar dust. Mon. Not. $R$. Astron. Soc. 1983, 205, 67-70.

(7) Sakata, A.; Wada, S.; Tanabe, T.; Onaka, T. Infrared spectrum of the laboratory-synthesized quenched carbonaceous composite (QCC) - Comparison with the infrared unidentified emission bands. Astrophys. J. 1984, 287, L51-L54.

(8) Allamandola, L. J.; Tielens, A. G. G. M.; Barker, J. R. Interstellar polycyclic aromatic hydrocarbons - The infrared emission bands, the excitation/emission mechanism, and the astrophysical implications. Astrophys. J., Suppl. Ser. 1989, 71, 733-775.

(9) Puget, J. L.; Léger, A. A new component of the interstellar matter - Small grains and large aromatic molecules. Annu. Rev. Astron. Astrophys. 1989, 27, 161.

(10) Jones, A. P.; d'Hendecourt, L. Interstellar nanodiamonds: the carriers of mid-infrared emission bands? Astron. Astrophys. 2000, 355, $1191-1200$

(11) Petrie, S.; Stranger, R.; Duley, W. W. Locally Aromatic Polycyclic Hydrocarbons as Potential Carriers of Infrared Emission Features. Astrophys. J. 2003, 594, 869-873.

(12) Kwok, S.; Zhang, Y. Mixed aromatic-aliphatic organic nanoparticles as carriers of unidentified infrared emission features. Nature 2011, 479, 80-83.

(13) PAH cluster refers to an aggregate of molecules, typically bound by weak van der Waals forces, with a size of up to 1000 atoms (e.g., $20 \mathrm{PAH}$ molecules). Beyond this size limit, the term very small grains (VSGs) is used in astronomy. In addition, some covalent bonding between the cluster or very small grains is sometimes considered likely.

(14) Cernicharo, J.; Heras, A. M.; Tielens, A. G. G. M.; Pardo, J. R.; Herpin, F.; Guélin, M.; Waters, L. B. F. M. Infrared Space Observatory's discovery of $\mathrm{C}_{4} \mathrm{H}_{2}, \mathrm{C}_{6} \mathrm{H}_{2}$, and benzene in CRL 618 . Astrophys. J. 2001, 546, L123-L126.

(15) McGuire, B. A.; Burkhardt, A. M.; Kalenskii, S.; Shingledecker, C. N.; Remijan, A. J.; Herbst, E.; McCarthy, M. C. Detection of the aromatic molecule benzonitrile $\left(\mathrm{c}^{-} \mathrm{C}_{6} \mathrm{H}_{5} \mathrm{CN}\right)$ in the interstellar medium. Science 2018, 359, 202-205.

(16) Cami, J.; Bernard-Salas, J.; Peeters, E.; Malek, S. E. Detection of $\mathrm{C}_{60}$ and $\mathrm{C}_{70}$ in a young planetary nebula. Science 2010, 329, 1180.

(17) Sellgren, K.; Werner, M. W.; Ingalls, J. G.; Smith, J. D. T.; Carleton, T. M.; Joblin, C. $\mathrm{C}_{60}$ in Reflection Nebulae. Astrophys. J., Lett. 2010, 722, L54-L57.

(18) Galliano, F.; Madden, S.; Tielens, A.; Peeters, E.; Jones, A. Variations of the mid-IR aromatic features inside and among galaxies. Astrophys. J. 2008, 679, 310.

(19) Boersma, C.; Bregman, J.; Allamandola, L. J. Properties of Polycyclic Aromatic Hydrocarbons in the Northwest Photon Dominated Region of NGC 7023. III. Quantifying the Traditional Proxy for PAH Charge and Assessing its Role. Astrophys. J. 2015, 806, 121.

(20) Stock, D. J.; Peeters, E. Polycyclic Aromatic Hydrocarbon Emission in Spitzer/IRS Maps. II. A Direct Link between Band Profiles and the Radiation Field Strength. Astrophys. J. 2017, 837, 129.

(21) Maragkoudakis, A.; Peeters, E.; Ricca, A. Probing the size and charge of polycyclic aromatic hydrocarbons. Mon. Not. R. Astron. Soc. 2020, 494, 642-664.

(22) Draine, B. T.; Li, A.; Hensley, B. S.; Hunt, L. K.; Sandstrom, K.; Smith, J. D. T. Excitation of PAH Emission: Dependence on Starlight Spectrum, Intensity, PAH Size Distribution, and PAH Ionization. 2020, https://arxiv.org/abs/2011.07046.

(23) Li, A. Spitzer's perspective of polycyclic aromatic hydrocarbons in galaxies. Nat. Astron. 2020, 4, 339-351.

(24) Calzetti, D. Star formation in galaxies as traced by the Spitzer Space Telescope. Nat. Astron. 2020, 4, 437-439.
(25) Peeters, E.; Allamandola, L. J.; Hudgins, D. M.; Hony, S.; Tielens, A. G. G. M. The Unidentified InfraRed Features after ISO. Astrophysics of Dust. Astron. Soc. Pac. Conf. Ser. 2004, 309, 141.

(26) Peeters, E.; Hony, S.; Van Kerckhoven, C.; Tielens, A. G. G. M.; Allamandola, L. J.; Hudgins, D. M.; Bauschlicher, C. W. The rich 6 to $9 \mu \mathrm{m}$ PAH spectrum. Astron. Astrophys. 2002, 390, 1089.

(27) van Diedenhoven, B.; Peeters, E.; Van Kerckhoven, C.; Hony, S.; Allamandola, L. J.; Hudgins, D. M.; Tielens, A. G. G. M. The profiles of the 3.3 to $12 \mu \mathrm{m}$ PAH features. Astrophys. J. 2004, 611, 928-939.

(28) Matsuura, M.; Bernard-Salas, J.; Lloyd Evans, T.; Volk, K. M.; Hrivnak, B. J.; Sloan, G. C.; Chu, Y.; Gruendl, R.; Kraemer, K. E.; Peeters, E.; Szczerba, R.; Wood, P. R.; Zijlstra, A. A.; Hony, S.; Ita, Y.; Kamath, D.; Lagadec, E.; Parker, Q. A.; Reid, W. A.; Shimonishi, T.; Van Winckel, H.; Woods, P. M.; Kemper, F.; Meixner, M.; Otsuka, M.; Sahai, R.; Sargent, B. A.; Hora, L. J.; McDonald, I. Spitzer Space Telescope spectra of post-AGB stars in the Large Magellanic Cloud polycyclic aromatic hydrocarbons at low metallicities. Mon. Not. $R$. Astron. Soc. 2014, 439, 1472-1493.

(29) Sloan, G. C.; Lagadec, E.; Zijlstra, A. A.; Kraemer, K. E.; Weis, A. P.; Matsuura, M.; Volk, K.; Peeters, E.; Duley, W. W.; Cami, J.; Bernard-Salas, J.; Kemper, F.; Sahai, R. Carbon-rich Dust Past the Asymptotic Giant Branch: Aliphatics, Aromatics, and Fullerenes in the Magellanic Clouds. Astrophys. J. 2014, 791, 28.

(30) Jensen, P. A.; Shannon, M. J.; Peeters, E.; Sloan, G. C.; Stock, D. J. The aliphatic bands in Polycyclic Aromatic Hydrocarbon emission. Astron. Astrophys., manuscript in preparation, 2021.

(31) Kerr, T. H.; Hurst, M. E.; Miles, J. R.; Sarre, P. J. Observations of the 3.3- $\mu \mathrm{m}$ UIR band in the Red Rectangle: relation to unidentified optical emission. Mon. Not. R. Astron. Soc. 1999, 303, 446-454.

(32) Candian, A.; Kerr, T. H.; Song, I.-O.; McCombie, J.; Sarre, P. J. Spatial distribution and interpretation of the $3.3 \mu \mathrm{m}$ PAH emission band of the Red Rectangle. Mon. Not. R. Astron. Soc. 2012, 426, 389397.

(33) Boersma, C.; Bauschlicher, C. W., Jr.; Ricca, A.; Mattioda, A. L.; Cami, J.; Peeters, E.; Sánchez de Armas, F.; Puerta Saborido, G.; Hudgins, D. M.; Allamandola, L. J. The NASA Ames PAH IR Spectroscopic Database Version 2.00: Updated Content, Web Site, and On(Off)line Tools. Astrophys. J., Suppl. Ser. 2014, 211, 8.

(34) Bernard-Salas, J.; Peeters, E.; Sloan, G. C.; Gutenkunst, S.; Matsuura, M.; Tielens, A. G. G. M.; Zijlstra, A. A.; Houck, J. R. Unusual Dust Emission from Planetary Nebulae in the Magellanic Clouds. Astrophys. J. 2009, 699, 1541-1552.

(35) Sloan, G. C.; Jura, M.; Duley, W. W.; Kraemer, K. E.; BernardSalas, J.; Forrest, W. J.; Sargent, B.; Li, A.; Barry, D. J.; Bohac, C. J.; Watson, D. M.; Houck, J. R. The Unusual Hydrocarbon Emission from the Early Carbon Star HD 100764: The Connection between Aromatics and Aliphatics. Astrophys. J. 2007, 664, 1144-1153.

(36) Bauschlicher, C. W., Jr.; Peeters, E.; Allamandola, L. J. The infrared spectra of very large, compact highly symmetric Polycyclic Aromatic Hydrocarbons (PAHs). Astrophys. J. 2008, 678, 316.

(37) Bauschlicher, C. W.; Peeters, E.; Allamandola, L. J. The Infrared Spectra of Very Large Irregular Polycyclic Aromatic Hydrocarbons (PAHs): Observational Probes of Astronomical PAH Geometry, Size, and Charge. Astrophys. J. 2009, 697, 311-327.

(38) Seok, J. Y.; Li, A. Polycyclic Aromatic Hydrocarbons in Protoplanetary Disks around Herbig Ae/Be and T Tauri Stars. Astrophys. J. 2017, 835, 291.

(39) Shannon, M. J.; Boersma, C. Examining the class B to A shift of the $7.7 \mu \mathrm{m}$ PAH band with the NASA Ames PAH IR Spectroscopic Database. Astrophys. J. 2019, 871, 214.

(40) Hudgins, D. M.; Bauschlicher, C. W., Jr.; Allamandola, L. J. Variations in the Peak Position of the $6.2 \mu \mathrm{m}$ Interstellar Emission Feature: A Tracer of $\mathrm{N}$ in the Interstellar Polycyclic Aromatic Hydrocarbon Population. Astrophys. J. 2005, 632, 316-332.

(41) Boersma, C.; Bouwman, J.; Lahuis, F.; van Kerckhoven, C.; Tielens, A. G. G. M.; Waters, L. B. F. M.; Henning, T. The characteristics of the IR emission features in the spectra of Herbig Ae 
stars: evidence for chemical evolution. Astron. Astrophys. 2008, 484, 241-249.

(42) Keller, L. D.; Sloan, G. C.; Forrest, W. J.; Ayala, S.; D’Alessio, P.; Shah, S.; Calvet, N.; Najita, J.; Li, A.; Hartmann, L.; Sargent, B.; Watson, D. M.; Chen, C. H. PAH Emission from Herbig Ae/Be Stars. Astrophys. J. 2008, 684, 411-429.

(43) Pino, T.; Dartois, E.; Cao, A.-T.; Carpentier, Y.; Chamaillé, T.; Vasquez, R.; Jones, A. P.; D’Hendecourt, L.; Bréchignac, P. The 6.2 $\mu \mathrm{m}$ band position in laboratory and astrophysical spectra: a tracer of the aliphatic to aromatic evolution of interstellar carbonaceous dust. Astron. Astrophys. 2008, 490, 665-672.

(44) Acke, B.; Bouwman, J.; Juhász, A.; Henning, T.; van den Ancker, M. E.; Meeus, G.; Tielens, A. G. G. M.; Waters, L. B. F. M. Spitzer's View on Aromatic and Aliphatic Hydrocarbon Emission in Herbig Ae Stars. Astrophys. J. 2010, 718, 558-574.

(45) Dartois, E.; Charon, E.; Engrand, C.; Pino, T.; Sandt, C. Mechanochemical synthesis of aromatic infrared band carriers. The top-down chemistry of interstellar carbonaceous dust grain analogues. Astron. Astrophys. 2020, 637, A82.

(46) Blasberger, A.; Behar, E.; Perets, H. B.; Brosch, N.; Tielens, A. G. G. M. Observational Evidence Linking Interstellar UV Absorption to PAH Molecules. Astrophys. J. 2017, 836, 173.

(47) Bregman, J. D.; Allamandola, L. J.; Witteborn, F. C.; Tielens, A. G. G. M.; Geballe, T. R. The infrared emission bands. II - A spatial and spectral study of the Orion bar. Astrophys. J. 1989, 344, 791-798.

(48) Joblin, C.; Tielens, A. G. G. M.; Geballe, T. R.; Wooden, D. H. Variations of the 8.6 and 11.3 MU M Emission Bands within NGC 1333: Evidence for PAH Cations. Astrophys. J., Lett. 1996, 460, L119. (49) Sloan, G. C.; Hayward, T. L.; Allamandola, L. J.; Bregman, J. D.; Devito, B.; Hudgins, D. M. Direct Spectroscopic Evidence for Ionized Polycyclic Aromatic Hydrocarbons in the Interstellar Medium. Astrophys. J. 1999, 513, L65-L68.

(50) Hudgins, D. M.; Allamandola, L. J. Interstellar PAH Emission in the 11-14 Micron Region: New Insights from Laboratory Data and a Tracer of Ionized PAHS. Astrophys. J. 1999, 516, L41.

(51) Allamandola, L. J.; Hudgins, D. M.; Sandford, S. A. Modeling the Unidentified Infrared Emission with Combinations of Polycyclic Aromatic Hydrocarbons. Astrophys. J. 1999, 511, L115-L119.

(52) Stock, D. J.; Choi, W. D.-Y.; Moya, L. G. V.; Otaguro, J. N.; Sorkhou, S.; Allamandola, L. J.; Tielens, A. G. G. M.; Peeters, E. Polycyclic Aromatic Hydrocarbon Emission in Spitzer/IRS Maps. I. Catalog and Simple Diagnostics. Astrophys. J. 2016, 819, 65.

(53) Whelan, D. G.; Lebouteiller, V.; Galliano, F.; Peeters, E.; Bernard-Salas, J.; Johnson, K. E.; Indebetouw, R.; Brandl, B. R. An Indepth View of the Mid-infrared Properties of Point Sources and the Diffuse ISM in the SMC Giant H II Region, N66. Astrophys. J. 2013, 771, 16.

(54) Sidhu, A.; Peeters, E.; Cami, J.; Knight, C. A principal component analysis of polycyclic aromatic hydrocarbon emission in NGC 2023. Mon. Not. R. Astron. Soc. 2020, 500, 177-190.

(55) Maragkoudakis, A.; Ivkovich, N.; Peeters, E.; Stock, D. J.; Hemachandra, D.; Tielens, A. G. G. M. PAHs and star formation in the H II regions of nearby galaxies M83 and M33. Mon. Not. R. Astron. Soc. 2018, 481, 5370-5393.

(56) Knight, C.; Peeters, E.; Wolfire, M. G.; Stock, D. J. PAH emission in the reflection nebula NGC 1333. Mon. Not. R. Astron. Soc., manuscript in preparation, 2021.

(57) Knight, C.; Peeters, E.; Stock, D. J.; Vacca, W. D.; Tielens, A. G. G. M. Tracing PAH size in prominent nearby mid-infrared environments. Astrophys. J., submitted for publication, 2021.

(58) Knight, C. Ph.D. Thesis, University of Western Ontario (Canada), 2020.

(59) Schutte, W. A.; Tielens, A. G. G. M.; Allamandola, L. J. Theoretical modeling of the infrared fluorescence from interstellar polycyclic aromatic hydrocarbons. Astrophys. J. 1993, 415, 397-414.

(60) Li, A.; Draine, B. T. Infrared Emission from Interstellar Dust. II. The Diffuse Interstellar Medium. Astrophys. J. 2001, 554, 778-802.

(61) Ricca, A.; Bauschlicher, C. W., Jr.; Boersma, C.; Tielens, A. G. G. M.; Allamandola, L. J. The Infrared Spectroscopy of Compact
Polycyclic Aromatic Hydrocarbons Containing up to 384 Carbons. Astrophys. J. 2012, 754, 75.

(62) Mori, T. I.; Sakon, I.; Onaka, T.; Kaneda, H.; Umehata, H.; Ohsawa, R. Observations of the near- to Mid-infrared Unidentified Emission Bands in the Interstellar Medium of the Large Magellanic Cloud. Astrophys. J. 2012, 744, 68.

(63) Croiset, B. A.; Candian, A.; Berné, O.; Tielens, A. G. G. M. Mapping PAH sizes in NGC 7023 with SOFIA. Astron. Astrophys. 2016, 590, A26.

(64) Sandstrom, K. M.; Bolatto, A. D.; Bot, C.; Draine, B. T.; Ingalls, J. G.; Israel, F. P.; Jackson, J. M.; Leroy, A. K.; Li, A.; Rubio, M.; Simon, J. D.; Smith, J. D. T.; Stanimirović, S.; Tielens, A. G. G. M.; van Loon, J. T. The Spitzer Spectroscopic Survey of the Small Magellanic Cloud $\left(\mathrm{S}^{4} \mathrm{MC}\right)$ : Probing the Physical State of Polycyclic Aromatic Hydrocarbons in a Low-metallicity Environment. Astrophys. J. 2012, 744, 20.

(65) Lai, T. S. Y.; Smith, J. D. T.; Baba, S.; Spoon, H. W. W.; Imanishi, M. All the PAHs: An AKARI-Spitzer Cross-archival Spectroscopic Survey of Aromatic Emission in Galaxies. Astrophys. J. 2020, 905, 55.

(66) Bellamy, L. The infra-red spectra of complex molecules, 2nd ed.; John Wiley \& Sons, Inc.: New York, 1958.

(67) Hony, S.; Van Kerckhoven, C.; Peeters, E.; Tielens, A. G. G. M.; Hudgins, D. M.; Allamandola, L. J. The CH out-of-plane bending modes of $\mathrm{PAH}$ molecules in astrophysical environments. Astron. Astrophys. 2001, 370, 1030.

(68) Shannon, M. J.; Stock, D. J.; Peeters, E. Interpreting the Subtle Spectral Variations of the 11.2 and $12.7 \mu \mathrm{m}$ Polycyclic Aromatic Hydrocarbon Bands. Astrophys. J. 2016, 824, 111.

(69) Mattioda, A. L.; Hudgins, D. M.; Bauschlicher, C. W.; Rosi, M.; Allamandola, L. J. Infrared Spectroscopy of Matrix-Isolated Polycyclic Aromatic Compounds and Their Ions. 6. Polycyclic Aromatic Nitrogen Heterocycles. J. Phys. Chem. A 2003, 107, 1486-1498.

(70) Weisman, J. L.; Mattioda, A.; Lee, T. J.; Hudgins, D. M.; Allamandola, L. J.; Bauschlicher, C. W., Jr.; Head-Gordon, M. Electronic transitions in the IR: Matrix isolation spectroscopy and electronic structure theory calculations on polyacenes and dibenzopolyacenes. Phys. Chem. Chem. Phys. 2005, 7, 109-118.

(71) Geballe, T. R.; Tielens, A. G. G. M.; Allamandola, L. J.; Moorhouse, A.; Brand, P. W. J. L. Spatial variations of the 3 micron emission features within UV-excited nebulae - Photochemical evolution of interstellar polycyclic aromatic hydrocarbons. Astrophys. J. 1989, 341, 278-287.

(72) Joblin, C.; Tielens, A. G. G. M.; Allamandola, L. J.; Geballe, T. R. Spatial Variation of the 3.29 and 3.40 Micron Emission Bands within Reflection Nebulae and the Photochemical Evolution of Methylated Polycyclic Aromatic Hydrocarbons. Astrophys. J. 1996, 458,610 .

(73) Sloan, G. C.; Bregman, J. D.; Geballe, T. R.; Allamandola, L. J.; Woodward, C. E. Variations in the 3 Micron Spectrum across the Orion Bar: Polycyclic Aromatic Hydrocarbons and Related Molecules. Astrophys. J. 1997, 474, 735.

(74) Pilleri, P.; Joblin, C.; Boulanger, F.; Onaka, T. Mixed aliphatic and aromatic composition of evaporating very small grains in NGC 7023 revealed by the $3.4 / 3.3 \mu \mathrm{m}$ ratio. Astron. Astrophys. 2015, 577, A16.

(75) Buss, R. H.; Cohen, M.; Tielens, A. G. G. M.; Werner, M. W.; Bregman, J. D.; Witteborn, F. C.; Rank, D.; Sandford, S. A. Hydrocarbon emission features in the IR spectra of warm supergiants. Astrophys. J. 1990, 365, L23-L26.

(76) Peeters, E.; Allamandola, L. J.; Bauschlicher, C. W., Jr.; Hudgins, D. M.; Sandford, S. A.; Tielens, A. G. G. M. Astrophys. J. 2004, 604, 252.

(77) Hudgins, D. M.; Bauschlicher, C. W., Jr.; Sandford, S. A. The Impact of Deuteration on the Infrared Spectra of Interstellar Polycyclic Aromatic Hydrocarbons. Astrophys. J. 2004, 614, 770-780.

(78) Doney, K. D.; Candian, A.; Mori, T.; Onaka, T.; Tielens, A. G. G. M. Deuterated polycyclic aromatic hydrocarbons: Revisited. Astron. Astrophys. 2016, 586, A65. 
(79) Joblin, C.; Boissel, P.; Leger, A.; D’Hendecourt, L.; Defourneau, D. Infrared spectroscopy of gas-phase PAH molecules. II. Role of the temperature. Astron. Astrophys. 1995, 299, 835.

(80) Jacox, M. E. The spectroscopy of molecular reaction intermediates trapped in the solid rare gases. Chem. Soc. Rev. 2002, $31,108-115$.

(81) Hudgins, D. M.; Allamandola, L. J. The Spacing of the Interstellar 6.2 and 7.7 Micron Emission Features as an Indicator of Polycyclic Aromatic Hydrocarbon Size. Astrophys. J. 1999, 513, L69.

(82) Sandford, S. A.; Bernstein, M. P.; Materese, C. K. The Infrared Spectra of Polycyclic Aromatic Hydrocarbons with Excess Peripheral $\mathrm{H}$ atoms $\left(\mathrm{H}_{n}-\mathrm{PAHs}\right)$ and Their Relation to the 3.4 and $6.9 \mu \mathrm{m}$ PAH Emission Features. Astrophys. J., Suppl. Ser. 2013, 205, 8.

(83) Mattioda, A. L.; Hudgins, D. M.; Boersma, C.; Bauschlicher, C. W.; Ricca, A.; Cami, J.; Peeters, E.; Sánchez de Armas, F.; Puerta Saborido, G.; Allamandola, L. J. The NASA Ames PAH IR Spectroscopic Database: The Laboratory Spectra. Astrophys. J., Suppl. Ser. 2020, 251, 22.

(84) Oomens, J.; Tielens, A. G. G. M.; Sartakov, B. G.; von Helden, G.; Meijer, G. Laboratory Infrared Spectroscopy of Cationic Polycyclic Aromatic Hydrocarbon Molecules. Astrophys. J. 2003, 591, 968-985.

(85) Knorke, H.; Langer, J.; Oomens, J.; Dopfer, O. Infrared Spectra of Isolated Protonated Polycyclic Aromatic Hydrocarbon Molecules. Astrophys. J. 2009, 706, L66-L70.

(86) Wiersma, S. D.; Candian, A.; Bakker, J. M.; Berden, G.; Eyler, J. R.; Oomens, J.; Tielens, A. G. G. M.; Petrignani, A. IR photofragmentation of the Phenyl Cation: Spectroscopy and Fragmentation Pathways. Phys. Chem. Chem. Phys. 2021, 23, 4334.

(87) Schlemmer, S.; Cook, D. J.; Harrison, J. A.; Wurfel, B.; Chapman, W.; Saykally, R. J. The Unidentified Interstellar Infrared Bands: PAHs as Carriers? Science 1994, 265, 1686-1689.

(88) Cook, D. J.; Schlemmer, S.; Balucani, N.; Wagner, D. R.; Harrison, J. A.; Steiner, B.; Saykally, R. J. Single photon infrared emission spectroscopy: a study of IR emission from UV laser excited PAHs between 3 and 15 um. J. Phys. Chem. A 1998, 102, 1465.

(89) Piest, H.; von Helden, G.; Meijer, G. Infrared Spectroscopy of Jet-cooled Cationic Polyaromatic Hydrocarbons: Naphthalene ${ }^{+}$. Astrophys. J. 1999, 520, L75-L78.

(90) Maltseva, E.; Petrignani, A.; Candian, A.; Mackie, C. J.; Huang, X.; Lee, T. J.; Tielens, A. G. G. M.; Oomens, J.; Buma, W. J. Highresolution IR absorption spectroscopy of polycyclic aromatic hydrocarbons: The realm of anharmonicity. Astrophys. J. 2015, 814, 23.

(91) Maltseva, E.; Petrignani, A.; Candian, A.; Mackie, C. J.; Huang, X.; Lee, T. J.; Tielens, A. G. G. M.; Oomens, J.; Buma, W. J. Highresolution IR absorption spectroscopy of polycyclic aromatic hydrocarbons in the $3 \mu \mathrm{m}$ region: role of periphery. Astrophys. J. 2016, 831, 58.

(92) Maltseva, E.; Mackie, C. J.; Candian, A.; Petrignani, A.; Huang, X.; Lee, T. J.; Tielens, A. G. G. M.; Oomens, J.; Buma, W. J. Highresolution IR absorption spectroscopy of polycyclic aromatic hydrocarbons in the $3 \mu \mathrm{m}$ region: role of hydrogenation and alkylation. Astron. Astrophys. 2018, 610, A65.

(93) Lemmens, A. K.; Rap, D. B.; Thunnissen, J. M. M.; Mackie, C. J.; Candian, A.; Tielens, A. G. G. M.; Rijs, A. M.; Buma, W. J. Anharmonicity in the mid-infrared spectra of polycyclic aromatic hydrocarbons: molecular beam spectroscopy and calculations. Astron. Astrophys. 2019, 628, A130.

(94) Ricca, A.; Bauschlicher, C. W., Jr.; Roser, J. E.; Peeters, E. Polycyclic Aromatic Hydrocarbons with Straight Edges and the 7.6/ 6.2 and 8.6/6.2 Intensity Ratios in Reflection Nebulae. Astrophys. J. 2018, 854, 115 .

(95) Malloci, G.; Joblin, C.; Mulas, G. On-line database of the spectral properties of polycyclic aromatic hydrocarbons. Chem. Phys. 2007, 332, 353-359.

(96) Bauschlicher, C. W., Jr.; Boersma, C.; Ricca, A.; Mattioda, A. L.; Cami, J.; Peeters, E.; Sánchez de Armas, F.; Puerta Saborido, G.; Hudgins, D. M.; Allamandola, L. J. The NASA Ames Polycyclic
Aromatic Hydrocarbon Infrared Spectroscopic Database: The Computed Spectra. Astrophys. J., Suppl. Ser. 2010, 189, 341-351.

(97) Bauschlicher, J.; Charles, W.; Ricca, A.; Boersma, C.; Allamandola, L. J. The NASA Ames PAH IR Spectroscopic Database: Computational Version 3.00 with Updated Content and the Introduction of Multiple Scaling Factors. Astrophys. J., Suppl. Ser. 2018, 234, 32.

(98) Bauschlicher, C. W.; Ricca, A. On the calculation of the vibrational frequencies of polycyclic aromatic hydrocarbons. Mol. Phys. 2010, 108, 2647-2654.

(99) Langhoff, S. R. Theoretical infrared spectra for polycyclic aromatic hydrocarbon neutrals, cations, and anions. J. Phys. Chem. 1996, 100, 2819-2841.

(100) Mackie, C. J.; Candian, A.; Huang, X.; Maltseva, E.; Petrignani, A.; Oomens, J.; Buma, W. J.; Lee, T. J.; Tielens, A. e. G. G. M. The anharmonic quartic force field infrared spectra of three polycyclic aromatic hydrocarbons: Naphthalene, anthracene, and tetracene. J. Chem. Phys. 2015, 143, 224314.

(101) Mackie, C. J.; Candian, A.; Huang, X.; Maltseva, E.; Petrignani, A.; Oomens, J.; Buma, W. J.; Lee, T. J.; Tielens, A. e. G. G. M. The anharmonic quartic force field infrared spectra of hydrogenated and methylated PAHs. Phys. Chem. Chem. Phys. 2018, 20, 1189-1197.

(102) Simon, A.; Rapacioli, M.; Lanza, M.; Joalland, B.; Spiegelman, F. Molecular dynamics simulations on $(\mathrm{FePAH})+\pi$-complexes of astrophysical interest: anharmonic infrared spectroscopy. Phys. Chem. Chem. Phys. 2011, 13, 3359-3374.

(103) Tielens, A. G. G. M. The Physics and Chemistry of the Interstellar Medium; Cambridge Univ. Press: Cambridge, U.K., 2005.

(104) Pirali, O.; Vervloet, M.; Mulas, G.; Malloci, G.; Joblin, C. High-resolution infrared absorption spectroscopy of thermally excited naphthalene. Measurements and calculations of anharmonic parameters and vibrational interactions. Phys. Chem. Chem. Phys. 2009, 11, 3443.

(105) Chakraborty, S.; Mulas, G.; Rapacioli, M.; Joblin, C. Modeling Anharmonic Infrared Spectra of Thermally Excited Pyrene $\left(\mathrm{C}_{16} \mathrm{H}_{10}\right)$ : the combined view of DFT AnharmonicCaOs and approximate DFT molecular dynamics. 2021, https://arxiv.org/abs/2102.06582.

(106) Pech, C.; Joblin, C.; Boissel, P. The profiles of the UIR bands explained with molecular carriers. Astron. Astrophys. 2002, 388, 639.

(107) Boersma, C.; Mattioda, A. L.; Bauschlicher, C. W.; Peeters, E.; Tielens, A. G. G. M.; Allamandola, L. J. The 5.25 and $5.7 \mu \mathrm{m}$ Astronomical Polycyclic Aromatic Hydrocarbon Emission Features. Astrophys. J. 2009, 690, 1208-1221.

(108) Le Page, V.; Snow, T. P.; Bierbaum, V. M. Hydrogenation and Charge States of Polycyclic Aromatic Hydrocarbons in Diffuse Clouds. II. Results. Astrophys. J. 2003, 584, 316-330.

(109) Bakes, E. L. O.; Tielens, A. G. G. M. The photoelectric heating mechanism for very small graphitic grains and polycyclic aromatic hydrocarbons. Astrophys. J. 1994, 427, 822-838.

(110) Verstraete, L.; Leger, A.; D’Hendecourt, L.; Defourneau, D.; Dutuit, O. Ionization cross-section measurements for two PAH molecules - Implications for the heating of diffuse interstellar gas. Astron. Astrophys. 1990, 237, 436-444.

(111) Jochims, H. W.; Baumgaertel, H.; Leach, S. Photoionization quantum yields of polycyclic aromatic hydrocarbons. Astron. Astrophys. 1996, 314, 1003-1009.

(112) Zhen, J.; Rodriguez Castillo, S.; Joblin, C.; Mulas, G.; Sabbah, H.; Giuliani, A.; Nahon, L.; Martin, S.; Champeaux, J.-P.; Mayer, P. M. VUV Photo-processing of PAH Cations: Quantitative Study on the Ionization versus Fragmentation Processes. Astrophys. J. 2016, $822,113$.

(113) Wenzel, G.; Joblin, C.; Giuliani, A.; Rodriguez Castillo, S.; Mulas, G.; Ji, M.; Sabbah, H.; Quiroga, S.; Peña, D.; Nahon, L. Astrochemical relevance of VUV ionization of large PAH cations. Astron. Astrophys. 2020, 641, A98.

(114) Sidhu, A.; Tielens, A. G. G. M.; Peeters, E.; Cami, J. Private communication, 2021. 
(115) Bakes, E. L. O.; Tielens, A. G. G. M. The Effects of Polycyclic Aromatic Hydrocarbons on the Chemistry of Photodissociation Regions. Astrophys. J. 1998, 499, 258-266.

(116) Wakelam, V.; Herbst, E. Polycyclic Aromatic Hydrocarbons in Dense Cloud Chemistry. Astrophys. J. 2008, 680, 371-383.

(117) Ge, J.; Mardones, D.; Inostroza, N.; Peng, Y. The roles of polycyclic aromatic hydrocarbons in dark cloud chemistry: new constraints on sulphur-bearing species. Mon. Not. R. Astron. Soc. 2020, 497, 3306-3322.

(118) Jochims, H. W.; Ruhl, E.; Baumgartel, H.; Tobita, S.; Leach, S. Size effects on dissociation rates of polycyclic aromatic hydrocarbon cations: Laboratory studies and astophysical implications. Astrophys. J. 1994, 420, 307-317.

(119) Tielens, A. G. G. M. Molecular astrophysics; Cambridge Univ. Press: Cambridge, U.K., 2021.

(120) Zhen, J.; Paardekooper, D. M.; Candian, A.; Linnartz, H.; Tielens, A. G. G. M. Quadrupole ion trap/time-of-flight photofragmentation spectrometry of the hexa-peri-hexabenzocoronene (HBC) cation. Chem. Phys. Lett. 2014, 592, 211-216.

(121) Berné, O.; Tielens, A. G. G. M. Formation of buckminsterfullerene $\left(\mathrm{C}_{60}\right)$ in interstellar space. Proc. Natl. Acad. Sci. U. S. A. 2012, 109, 401-406.

(122) Zhen, J.; Castellanos, P.; Paardekooper, D. M.; Linnartz, H.; Tielens, A. G. G. M. Laboratory Formation of Fullerenes from PAHs: Top-down Interstellar Chemistry. Astrophys. J., Lett. 2014, 797, L30. (123) Yang, X. J.; Li, A.; Glaser, R. Superhydrogenated Polycyclic Aromatic Hydrocarbon Molecules: Vibrational Spectra in the Infrared. Astrophys. J., Suppl. Ser. 2020, 247, 1.

(124) Cazaux, S.; Boschman, L.; Rougeau, N.; Reitsma, G.; Hoekstra, R.; Teillet-Billy, D.; Morisset, S.; Spaans, M.; Schlathölter, T. The sequence to hydrogenate coronene cations: A journey guided by magic numbers. Sci. Rep. 2016, 6, 19835.

(125) Jensen, P. A.; Leccese, M.; Simonsen, F. D. S.; Skov, A. W.; Bonfanti, M.; Thrower, J. D.; Martinazzo, R.; Hornekær, L. Identification of stable configurations in the superhydrogenation sequence of polycyclic aromatic hydrocarbon molecules. Mon. Not. $R$. Astron. Soc. 2019, 486, 5492-5498.

(126) Berné, O.; Montillaud, J.; Joblin, C. Top-down formation of fullerenes in the interstellar medium. Astron. Astrophys. 2015, 577, A133. 